\title{
TorsinA hypofunction causes abnormal twisting movements and sensorimotor circuit neurodegeneration
}

\author{
Chun-Chi Liang, ${ }^{1}$ Lauren M. Tanabe, ${ }^{1}$ Stephanie Jou, ${ }^{1}$ Frank Chi, ${ }^{1}$ and William T. Dauer ${ }^{1,2}$ \\ ${ }^{1}$ Department of Neurology and 2Department of Cell and Developmental Biology, University of Michigan Medical School, Ann Arbor, Michigan, USA.
}

\begin{abstract}
Lack of a preclinical model of primary dystonia that exhibits dystonic-like twisting movements has stymied identification of the cellular and molecular underpinnings of the disease. The classical familial form of primary dystonia is caused by the DYT1 $(\triangle \mathrm{E})$ mutation in TOR $1 \mathrm{~A}$, which encodes torsin A, $\mathrm{AAA}^{+} \mathrm{ATPase}$ resident in the lumen of the endoplasmic reticular/nuclear envelope. Here, we found that conditional deletion of Tor $1 a$ in the CNS (nestin-Cre Tor $1 a^{\text {flox/-) }}$ or isolated CNS expression of DYT1 mutant torsinA (nestin-Cre Tor $1 a^{\text {flox/ }} \Delta E$ ) causes striking abnormal twisting movements. These animals developed perinuclear accumulation of ubiquitin and the E3 ubiquitin ligase HRD1 in discrete sensorimotor regions, followed by neurodegeneration that was substantially milder in nestin-Cre Tor $1 a^{f l o x} / \Delta E$ compared with nestin-Cre Tor $1 a^{f l o x /}$ animals. Similar to the neurodevelopmental onset of DYT1 dystonia in humans, the behavioral and histopathological abnormalities emerged and became fixed during CNS maturation in the murine models. Our results establish a genetic model of primary dystonia that is overtly symptomatic, and link torsinA hypofunction to neurodegeneration and abnormal twisting movements. These findings provide a cellular and molecular framework for how impaired torsinA function selectively disrupts neural circuits and raise the possibility that discrete foci of neurodegeneration may contribute to the pathogenesis of DYT1 dystonia.
\end{abstract}

\section{Introduction}

The primary dystonias, a group of sporadic and inherited illnesses, are a striking example of selective vulnerability of CNS sensorimotor circuits $(1,2)$. Dystonia - defined as prolonged, involuntary twisting movements - is traditionally classified as "primary" if it occurs in isolation and in the absence of neuropathological change. In contrast, "secondary" dystonic movements occur consequent to CNS damage (e.g., from stroke, trauma, or neurodegeneration), and are typically accompanied by additional neurological signs and symptoms. This classification scheme has been criticized (3), however, because neuroimaging of patients with primary dystonia indicates the presence of subtle neuropathological changes, and few primary dystonia brains have been comprehensively analyzed (4). Indeed, despite considerable interest in the pathophysiology of primary dystonia, the molecular and cellular underpinnings of CNS dysfunction and the identity of affected motor structures and cell types remain poorly understood.

DYT1 dystonia is a common inherited form of primary dystonia that typically manifests during a discrete period of childhood, implicating abnormal development as the cause of motor dysfunction. This neurodevelopmental disease is caused by a $3 \mathrm{bp}$ in-frame mutation in TOR1A that removes a single glutamic acid residue $(\triangle \mathrm{E})$ from the torsinA protein. Torsin A is a ubiquitously expressed $\mathrm{AAA}^{+}$ protein residing in the lumen of the ER/nuclear envelope (ER/NE) space (5-7). Accumulating evidence indicates that the DYT1 mutation acts by impairing torsin $\mathrm{A}$ function through multiple mechanisms. These mechanisms include impairing binding to LAP1 and LULL1, proteins required for its enzymatic activity, and decreasing the levels of torsinA protein - an effect observed in skin fibroblasts from DYT1 subjects and tissue from DYT1 knockin mice (8-10).

Conflict of interest: The authors have declared that no conflict of interest exists. Citation for this article: J Clin Invest. 2014;124(7):3080-3092. doi:10.1172/JCI72830.
$\mathrm{AAA}^{+}$proteins function as multimers, and additional evidence suggests that disease mutant torsinA may also act via a dominantnegative mechanism $(7,11,12)$. The biological role of torsinA is not well understood, but it has been implicated most strongly in protein quality control (13-16) and nuclear membrane-localized functions $(7,8,17-19)$, including regulation of the LINC complex $(20,21)$.

Mouse genetic studies have established that Tor $1 a^{\Delta \mathrm{E}}$ is a loss-offunction allele (8). However, it has not been possible to connect the molecular and cellular consequences of torsinA dysfunction to dystonic movements, as mouse models based on the manipulation of Torla (or other primary dystonia-causing genes) do not exhibit overt, abnormal movements. Overexpression of DYT1 mutant torsinA in mice causes modest abnormalities in measures of beam walking, pawprint analysis, and motor learning, but not abnormal movements similar to those in patients with DYT1 dystonia (22-24). DYT1 torsinA transgenic rats reportedly show hind limb clasping during tail suspension and abnormalities in the pawprint analysis (25). Mice heterozygous for DYT1 knockin $\left(\operatorname{Tor} 1 a^{+/ \Delta \mathrm{E}}\right)$ or $\mathrm{KO}\left(\operatorname{Tor} 1 a^{+}{ }^{+}\right)$alleles also appear normal; reports differ as to whether these animals exhibit defects in a beam-walking task $(26,27)$. In contrast, homozygous knockin (Tor $\left.1 a^{\Delta \mathrm{E} / \Delta \mathrm{E}}\right)$ or $\mathrm{KO}$ $\left(\right.$ Tor $\left.1 a^{-1}\right)$ mice die shortly after birth (8). To overcome this early lethality, torsinA has been conditionally deleted from striatum, striatal cholinergic interneurons, cortical glutamatergic neurons, or cerebellar Purkinje cells (28-30). Similar to previous mutants, however, these animals appear grossly normal, exhibiting alterations in beam walking or open field behavior.

We speculated that previous torsinA transgenic or Torla mutant mice lack abnormal movements because none feature torsinA deficiency in the key structures during postnatal CNS development. To test this notion, we generated a series of conditional Torla mutants in which the entire CNS either lacks torsinA or expresses pathogenic $\Delta \mathrm{E}$-torsinA exclusively (e.g., CNS genotype is Tor1 $a^{\Delta \mathrm{E} /}$ ). These 
A

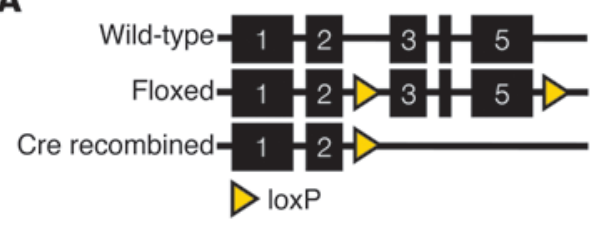

C

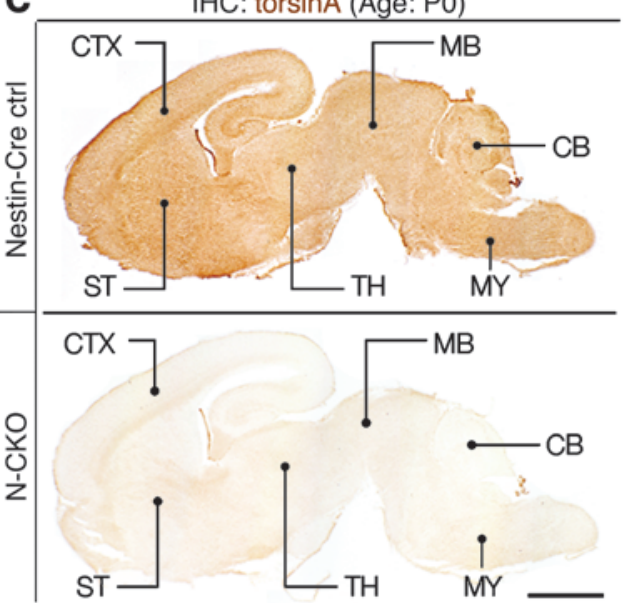

E
B

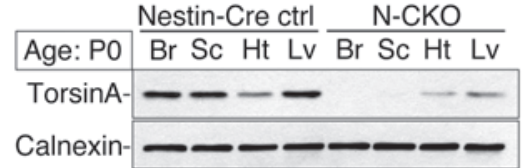

D

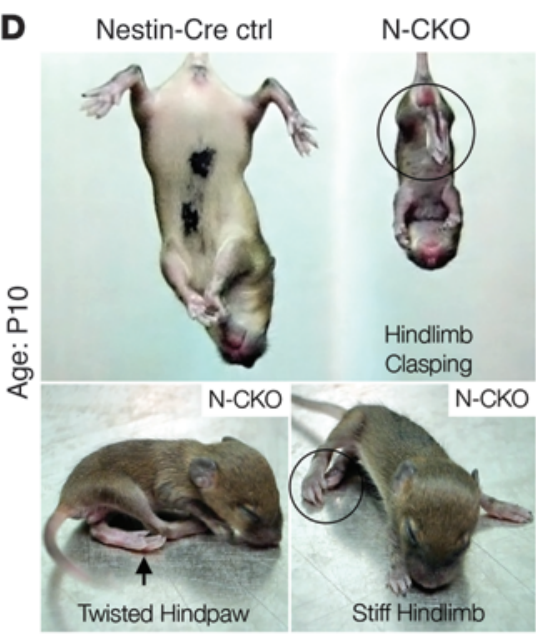

F

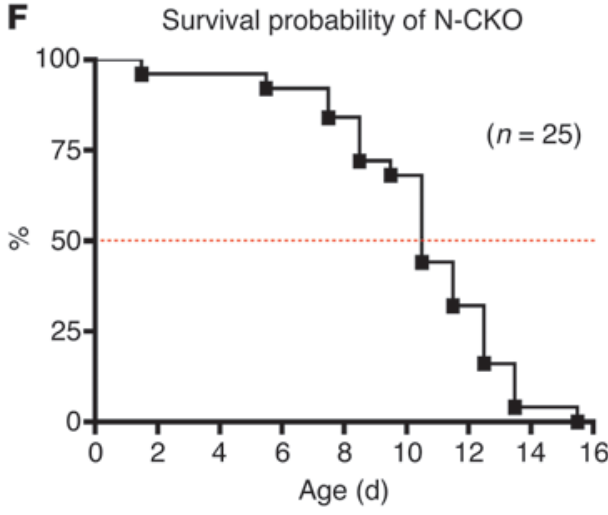

\section{Figure 1}

Conditional CNS deletion of torsinA causes abnormal twisting and early lethality. (A) Design of Tor1a floxed and Cre-recombined alleles. LoxP sites are located upstream of exon 3 and downstream of exon 5. Cre recombination excises exons 3-5. (B) N-CKO mice lack torsinA expression in CNS. The ER protein calnexin is used as the loading control. Br, brain; Sc, spinal cord; Ht, heart; Lv, liver. (C) TorsinA immunoreactivity in PO brains of control and N-CKO mice. nestin-Cre ctrl: Tor1 $a^{\text {flox/++; }}$ nestin-Cre. CTX, cerebral cortex; ST, striatum; TH, thalamus; MB, midbrain; CB, cerebellum; MY, medulla. Scale bar: $1 \mathrm{~mm}$. (D) N-CKO mice exhibit prolonged abnormal twisting movements (also see Supplemental Video 1). (E) Growth curves of N-CKO cohort. N-CKO mice become significantly lighter than littermate controls from P3 onward. 2-way ANOVA was used. Data are shown as mean \pm SD. ${ }^{*} P<0.05 ;{ }^{* \star} P<0.001$. (F) Kaplan-Meier survival analysis of N-CKO mice. N-CKO mice were born in Mendelian ratios. 50\% of N-CKO mice died by P10. No N-CKO mice survived beyond P16.

are the first Tor1a mutants, to our knowledge, to exhibit overt dystonic-like twisting movements and show histopathologic change and defects of protein quality control localized to a group of sensorimotor structures. Our results establish an overtly symptomatic genetic model of primary dystonia and provide new insight into the circuitry and molecular pathophysiology of the disease.

\section{Results}

Conditional CNS deletion of torsin A causes abnormal twisting movements and neurodegeneration in a discrete set of sensorimotor structures. Constitutive Tor 1a KO mutants die at P0, preventing study of the effects of and $7 \mathrm{~N}$ (Figure 1, B and C). Gliosis was also observed in spinal cord (Supplemental Figure 1C). Activated caspase-3 immunostaining and several markers of increased ER stress were also observed selectively in the same regions and emerged with a time course similar to gliosis (Figure 2D, Supplemental Figure 1C, and Supplemental Figure 2). Moreover, several regions and cell types implicated from studies of secondary dystonia appeared normal, including inferior olive, striatum, and cerebellar cortex (including Purkinje cells). These data suggest that, during postnatal maturation, a discrete set of sensorimotor circuit structures exhibit selective vulnerability to torsinA loss of function. The data do not establish whether these structures 

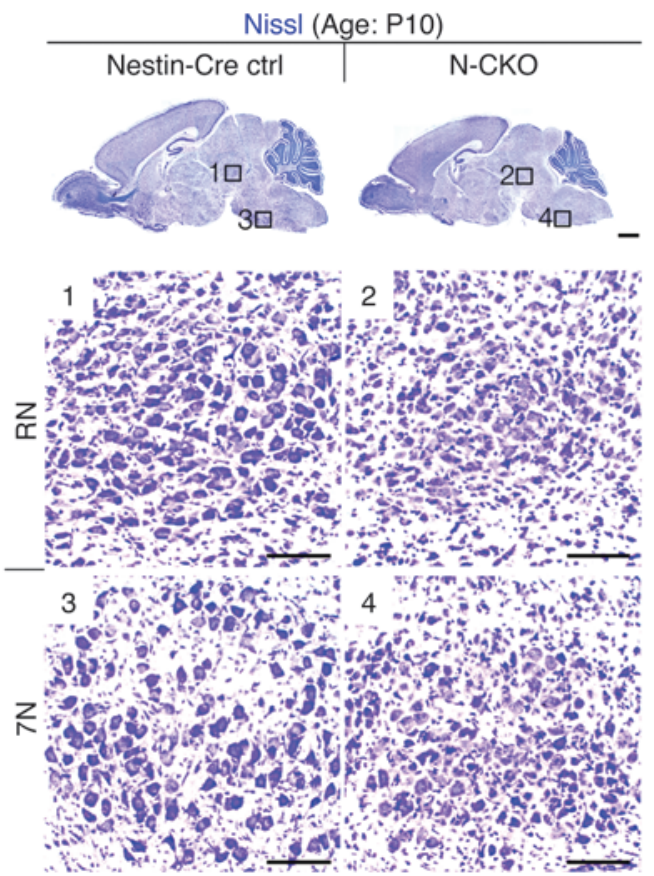

C

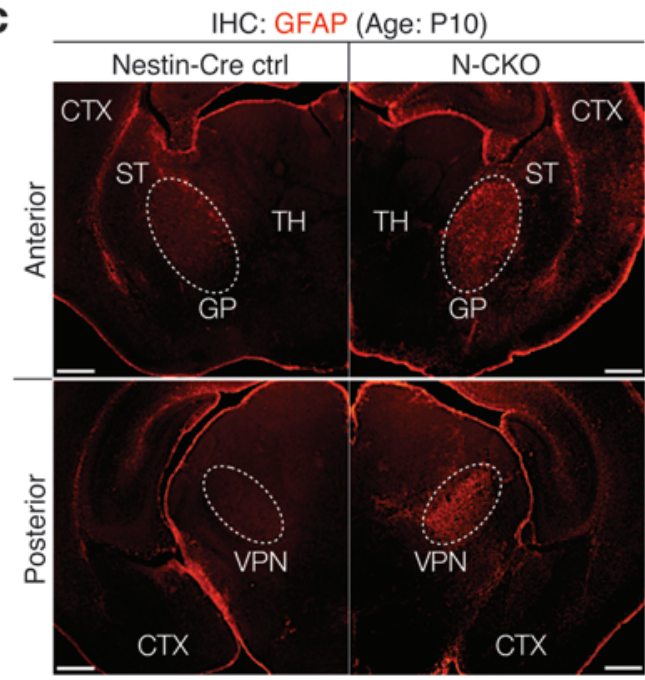

B

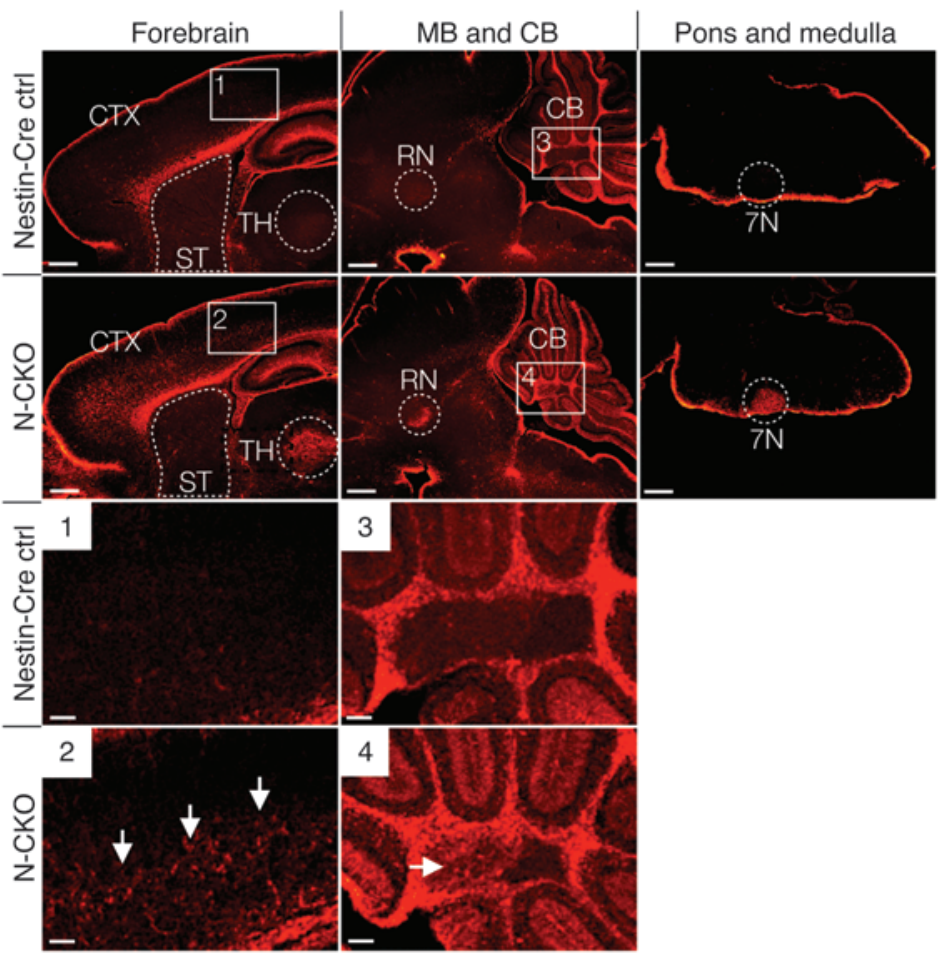

D

IHC: AC3 + Methyl green (N-CKO, Age: P10)

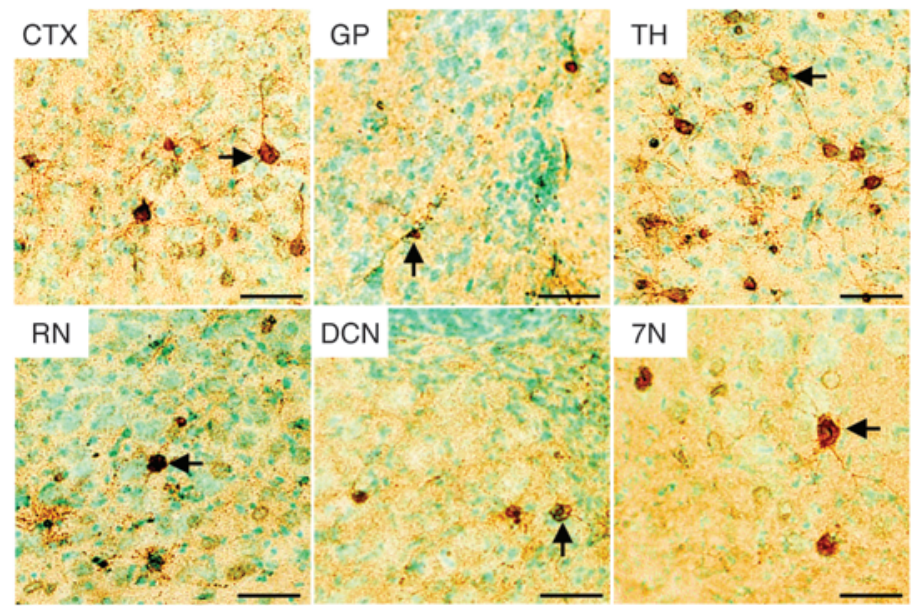

Figure 2

Conditional CNS deletion of torsinA causes sensorimotor circuit selective neurodegeneration. (A) Nissl stain of P10 control and N-CKO brains. High-magnification images of RN (insets 1 and 2) and 7N (insets 3 and 4) show loss of large neuronal cell bodies in N-CKO tissue. Scale bars: $1 \mathrm{~mm}$ (top panel, low-magnification, sagittal brain sections); $100 \mu \mathrm{m}$ (insets 1-4). (B) GFAP immunoreactivity in control and N-CKO brains at P10. N-CKO brains exhibit reactive gliosis selectively in deep cortical layers, GP (seen in C), ventral posterior thalamus, DCN, RN, and 7N. Highmagnification images from nestin-Cre ctrl (insets 1 and 3) and N-CKO (insets 2 and 4) highlight selective gliosis in cerebral cortex and DCN. Scale bars: $500 \mu \mathrm{m}$ (top panel, low-magnification, sagittal brain sections); $100 \mu \mathrm{m}$ (insets 1-4). (C) Coronal sections of N-CKO brains showing gliosis in GP and ventral posterior nucleus of the thalamus (VPN). Scale bars: $500 \mu \mathrm{m}$. (D) Immunoreactivity of activated caspase-3 (AC3) in P10 $\mathrm{N}-\mathrm{CKO}$ brains. Arrows highlight one of many positive cells in each vulnerable region. Scare bars: $50 \mu \mathrm{m}$.

are uniquely vulnerable to torsinA-related neurodegeneration, however, as the early death of $\mathrm{N}-\mathrm{CKO}$ prevents the analysis of additional regions that could become affected at later ages.

TorsinA-related neurodegeneration is region autonomous and becomes fixed during early postnatal development. The occurrence of selective neurodegeneration in multiple sensorimotor structures led us to ask whether this pattern of damage might arise by progression through a circuit via a non-cell autonomous mechanism. Conditional deletion of tor$\sin \mathrm{A}$ from neural progenitor cells that give rise to cortex (Emx1-Cre) or midbrain/hindbrain (En1-Cre), however, caused degeneration exclusively within the respective Cre expression fields (Figure 3). Within each Cre expression field, neurodegeneration remained lim- 


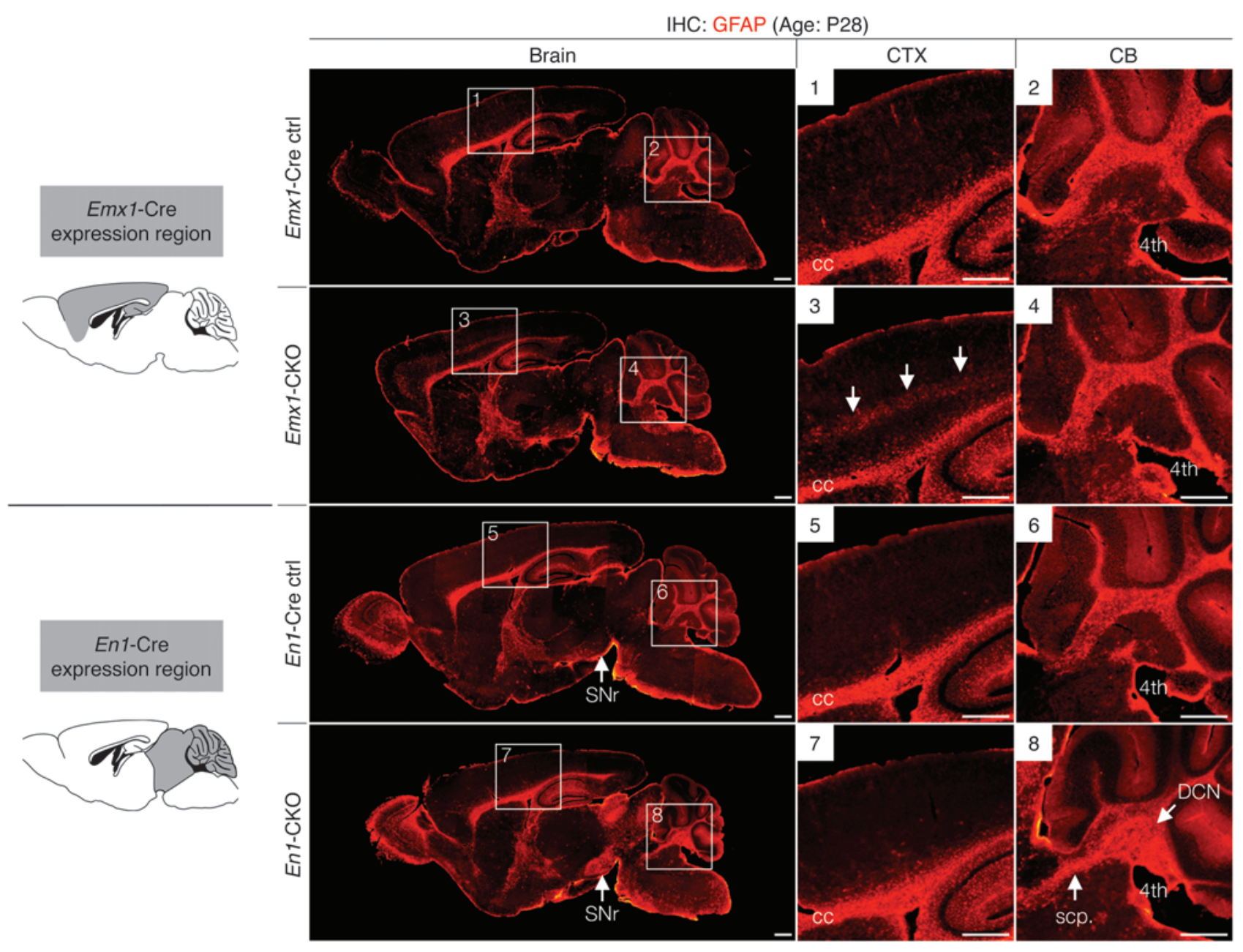

\section{Figure 3}

TorsinA-mediated neurodegeneration remains confined to Cre-expressing brain regions. Tor1a was deleted from neural progenitor cell (NPC), giving rise to cortex (Emx1-Cre) or midbrain/hindbrain (En1-Cre). Note that gliosis remains confined to previously defined selective regions that fall within the Cre-expressing region. SNr, substantia nigra pars reticulata; cc, corpus callosum; 4th, 4th ventricle; scp., superior cerebellar peduncle. Scare bars: $500 \mu \mathrm{m}$. High-magnification images from Emx1-Cre ctrl (insets 1 and 2), Emx1-CKO (insets 3 and 4), En1-Cre ctrl (insets 5 and 6), and $E n 1-C K O$ (insets 7 and 8 ) show selective reactive gliosis in deep cortical layers (Emx1-CKO) and DCN (En1-CKO).

ited to regions affected in $\mathrm{N}-\mathrm{CKO}$ mice, apart from the addition of substantia nigra pars reticulata at P15 (in En1-CKO mice) (Supplemental Figure 3, A and B). These conditional mutants exhibited normal viability, allowing assessment of the selectivity of neurodegeneration in mature mice. Gliosis remained restricted to the regions described above at up to 6 months of age (Supplemental Figure 3C). Collectively, these data demonstrate that a discrete set of sensorimotor structures are uniquely vulnerable to neurodegeneration caused by torsinA loss of function. In contrast to age-related neurodegeneration, which begins focally but typically spreads over time, the vulnerability of torsinA-sensitive regions appears to be cell autonomous and restricted, becoming fixed during CNS maturation.

Isolated expression of DYT1 mutant torsinA in neural progenitor cellsyields a robust model of DYT1 dystonia featuring overt twisting movements. We reported previously (31) that diffusion tensor imaging (DTI) of constitutive DYT1 heterozygous knockin mice shows a pattern of selective microstructural abnormalities that includes several brain regions identified as uniquely torsinA sensitive in N-CKO mice (Figure 2). Those animals were behaviorally normal (27), however, and showed no evidence of histopathological change when assessed by Nissl stain or immunostaining for GFAP or activated caspase-3 (data not shown). To further explore the disease relevance of the torsinA-sensitive regions identified in $\mathrm{N}-\mathrm{CKO}$ mice, we mimicked the presumed dominant negative behavior of the DYT1 mutation by generating mice carrying 1 DYT1 allele and 1 WT floxed allele, and conditionally deleted the WT floxed allele from the CNS with nestin-Cre (nestinCre $\operatorname{Tor} 1 a^{f l o x} / \Delta E$, herein referred to as nestin selective knockin [N-SKI]; Figure 4, A and B). This strategy recapitulated the non-cell type-specific abnormal concentration of torsinA in the NE (Figure 4C), as observed in primary fibroblasts from DYT1 patients (7). In contrast to N-CKO mice, N-SKI mice are fully viable, though significantly smaller than their littermate controls (Supplemental Figure 4A). $\mathrm{N}$-SKI mice initially had motoric function indistinguishable from that of their littermate controls, but during the second postnatal week developed spontaneous, overt abnormal movements and postures (Figure 4D, Supplemental Figure 4B, and Supplemental Video 2), including prolonged limb extension, abnormal toe position and tail extension, and action-induced forepaw clenching during tail sus- 


\section{A}

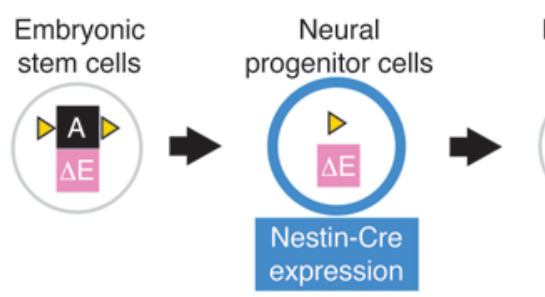

C

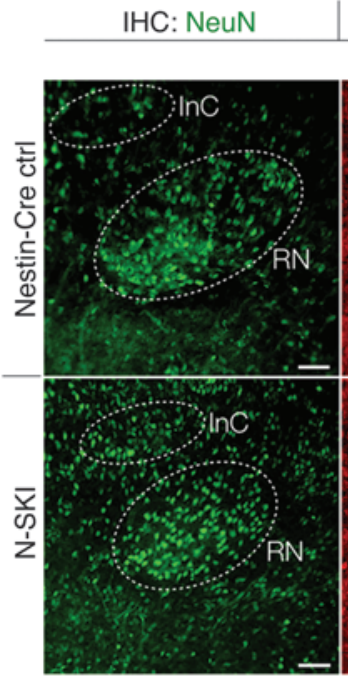

D
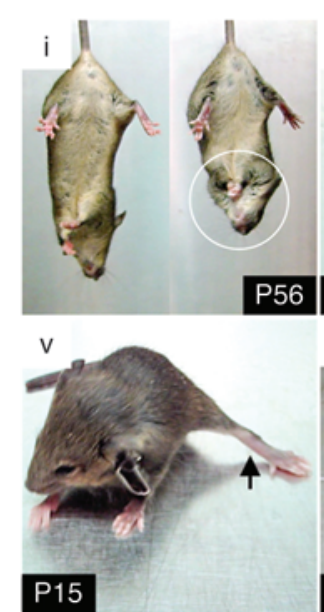

Neurons

and glia

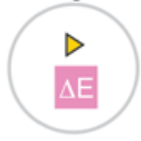

B

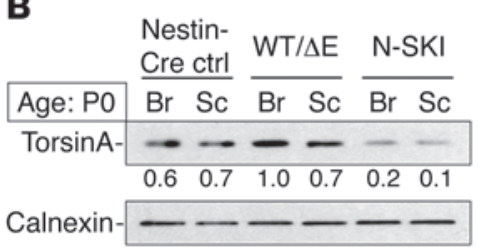

\section{Figure 4}

Isolated CNS expression of DYT1 mutant torsinA yields an overtly symptomatic model of DYT1 dystonia. (A) Nestin-Cre driven ablation of floxed torsinA results in isolated CNS expression of $\Delta \mathrm{E}$-torsin $\mathrm{A}(\Delta \mathrm{E})$ in the developing CNS. These animals are referred to as N-SKI. (B) Immunoblotting of torsinA expression in $\mathrm{PO}$ $\mathrm{N}-\mathrm{SKI}$ CNS. ER protein calnexin is used as the loading control. The numbers below the torsin A immunoblot represent the relative expression of torsinA normalized to those of $W T / \Delta E$. (C) TorsinA immunoreactivity in PO N-SKI brains shows abnormal perinuclear accumulation of $\Delta \mathrm{E}$-torsin $\mathrm{A}$ (insets 2 and 4). InC, interstitial nucleus of Cajal. Scale bars: $100 \mu \mathrm{m}$. (D) N-SKI mice exhibit several motor abnormalities, including (i) forelimb clasping, (ii) forepaw clenching, (iii) unilateral twisted hind paw, (iv) bilateral twisted hind paws, (v) prolonged stiff extension of hind limbs, (vi) abnormal toe postures, (vii) squinty eye(s), and (viii) tremors (in Supplemental Video 2). pension. Brain structural analysis by Nissl stain was unremarkable at birth (Supplemental Figure 4C), but at P56 showed loss of RN (Figure 5A). Unlike N-CKO mice, $7 \mathrm{~N}$ appeared largely spared (Figure 5A). $\mathrm{N}$-SKI mice recapitulated the pattern of gliotic change observed in N-CKO mice (Figure 5, B and C). The extent of gliosis, however, appeared less pronounced in all regions, especially in the spinal cord, which was minimally affected (Supplemental Figure 4D). Comparison of the number of activated caspase- 3 immunoreactive neurons confirmed that N-SKI mice exhibit significantly less histopathological change than N-CKO animals (Figure 5D). These data link the DYT1 mutation to brain regions that are selectively vulnerable to torsinA loss of function-related neurodegeneration.

The $\Delta E$ mutation causes neurodegeneration and abnormal twisting movements by impairing torsin A function. DTI neuroimaging of DYT1 subjects (32) and heterozygous DYT1 knockin mice (31) showed several abnormalities of hindbrain pathways, including in projections from the DCN that form the cerebellothalamic tract. To explore further the role of hindbrain structures in the motor phenotype of N-CKO and N-SKI mice, we compared the effects of conditional torsinA deletion or selective DYT1 mutant torsinA expression in these structures. We accomplished this by using En1-Cre transgenic mice to generate cohorts of En1 conditional KOs (En1-CKO; as in Figure 3 and Supplemental Figure 3) and En1 selective knockin (En1-SKI) mutants (Figure 6, A and B) and comparing the histopathological and behavioral features of these animals. Neuropathological analyses of these mutants with Nissl stain and immunostaining for GFAP showed that both exhibited abnormalities selectively in the hindbrain structures identified in N-CKO and N-SKI mice (Figure 6C). Using unbiased stereology in mature mice ( 2 months old), we found significant decreases in neuron number in the RN and DCN in both En1-CKO and En1-SKI mice (compared with littermate controls) (Figure 6, D and $\mathrm{E})$. However, while the degree of RN cell loss was similar in both 
A
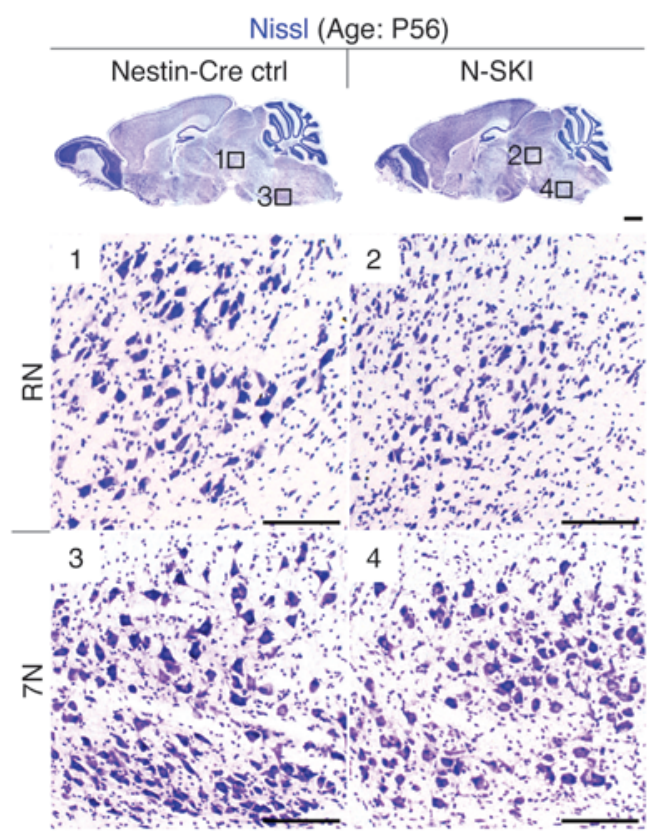

C

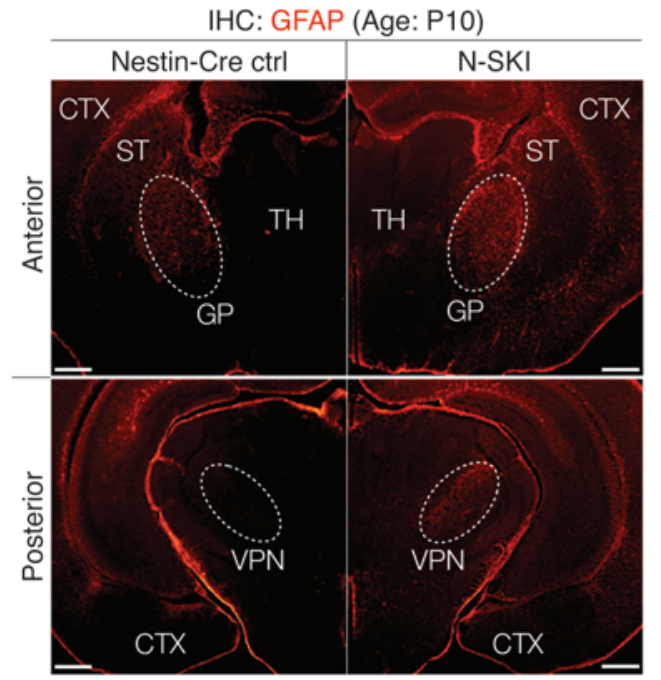

B
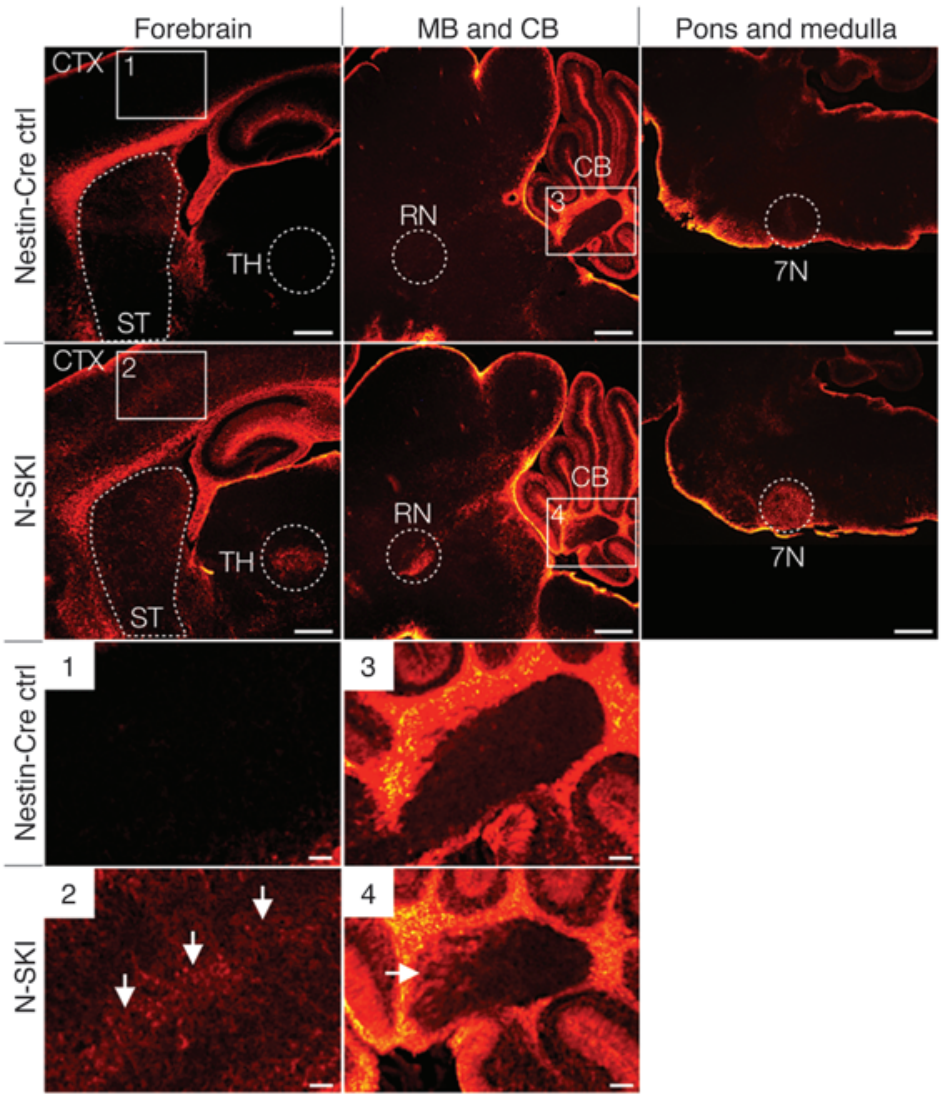

D

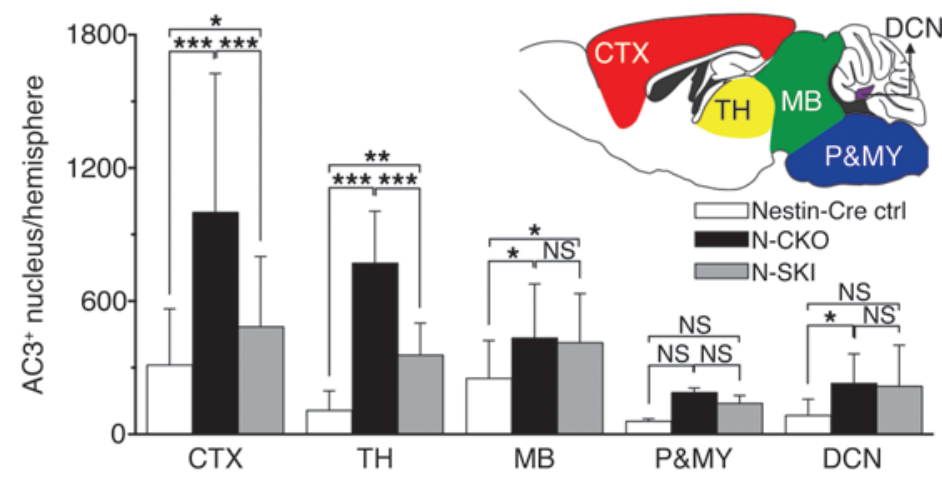

Figure 5

N-SKI mice exhibit sensorimotor circuit selective neurodegeneration. (A) Nissl analysis of P56 nestin-Cre ctrl and N-SKI brains. High-magnification images of RN (insets 1 and 2) and 7N (insets 3 and 4) show clear loss of large cell bodies in RN and partial loss in 7N. Scale bars: 1 mm (top panel, low-magnification, sagittal brain sections); $100 \mu \mathrm{m}$ (insets 1-4). (B) GFAP immunoreactivity (P10). Gliosis is present selectively in the regions identified as selectively vulnerable in N-CKO mice. High-magnification images from nestin-Cre ctrl (insets 1 and 3 ) and $\mathrm{N}-\mathrm{SKI}$ (insets 2 and 4) highlight reactive gliosis in cerebral cortex and DCN. Scale bars: $500 \mu \mathrm{m}$ (top panels, low-magnification, sagittal brain sections; $100 \mu \mathrm{m}$ (insets 1-4). (C) Coronal sections of N-SKI brains exhibit reactive gliosis in GP and ventral posterior nucleus (VPN). Scale bars: $500 \mu \mathrm{m}$. (D) Quantification of activated caspase-3-positive nuclei in right hemispheres of P10 nestin-Cre ctrl, N-CKO, and N-SKI. $n=4$. P, pons. 2-way ANOVA. Data are shown as mean \pm SD. ${ }^{*} P<0.05$; ${ }^{* *} P<0.01$; ${ }^{* *} P<0.001$.

mutants, DCN cell loss was roughly 2-fold greater in En1-CKO compared with En1-SKI mice (Figure 6, D and E). These data suggest that $\mathrm{RN}$ neurons may be more sensitive to torsinA loss of function than DCN neurons. In both mutants, the histopathological and behavioral defects emerged and became fixed during early postnatal development, with no apparent progression in adult animals.

We next compared the behavioral effects of torsinA loss of function and isolated expression of DYT1 mutant torsinA. En1-CKO and
En1-SKI mutants were born in Mendelian ratios and were indistinguishable from their control littermates at $\mathrm{P0}$ (data not shown). Both mutants were fully viable, but by P28, En1-CKO mice became significantly lighter than their littermate controls (Figure 7, A and B). All En1-CKO and En1-SKI mutants were abnormal in all tail-suspension indices at P15 (Figure 7, C-E). During tail suspension, both mutants exhibited hind limb and forelimb clasping, forepaw clenching, and twisted truncal postures. But with maturity, the forepaw clenching 
A

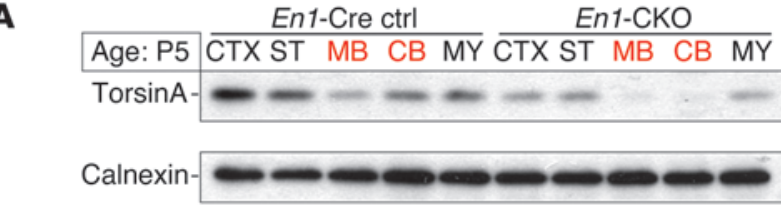

C
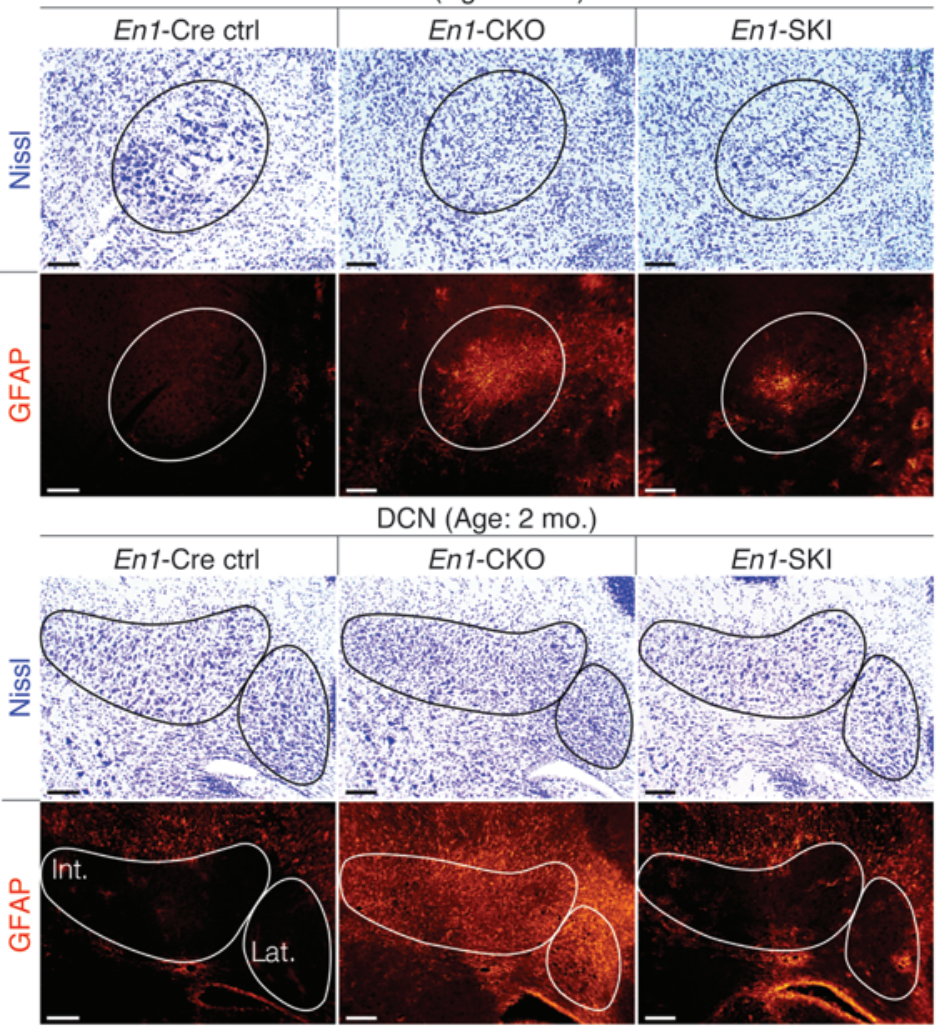

DCN (Age: 2 mo.)

RN (Age: 2 mo.)

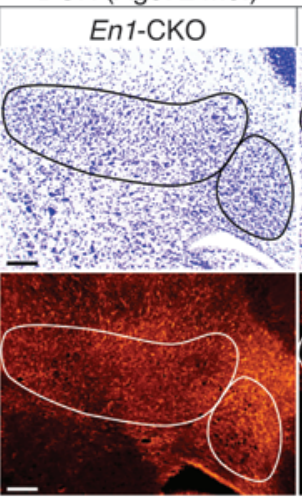

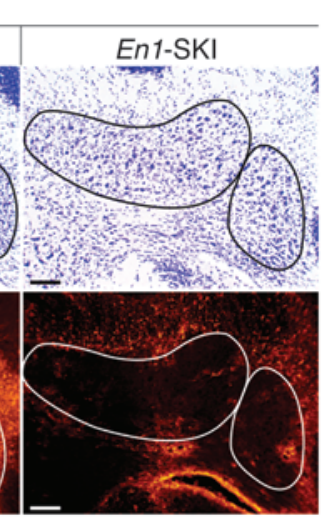

B

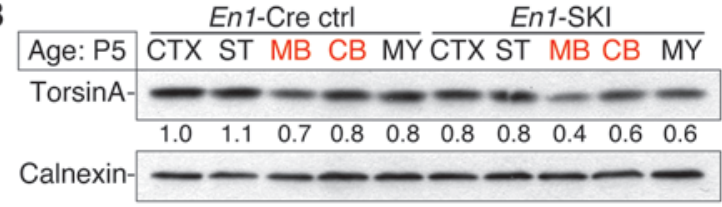

D

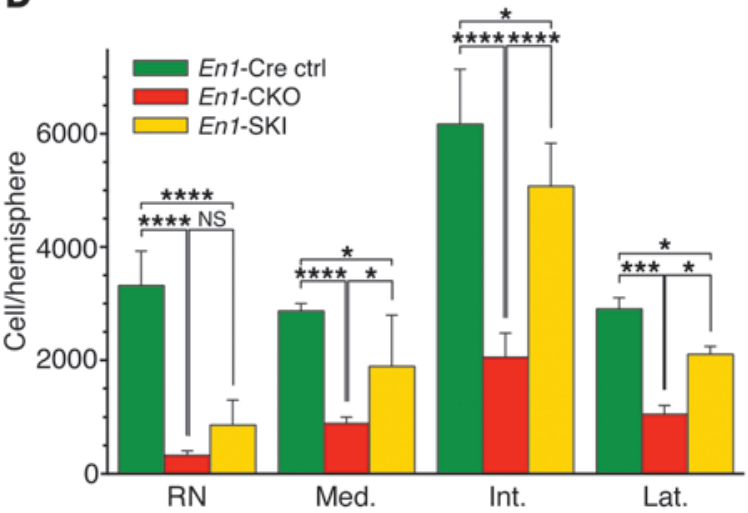

$\mathbf{E}$

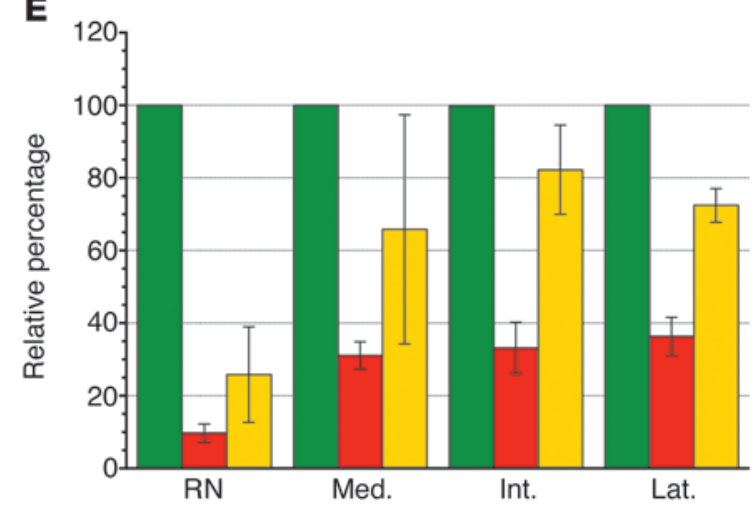

Figure 6

Characterization of RN and DCN neurodegeneration in adult En1-CKO and En1-SKI mice. (A) Immunoblotting of torsinA expression in P5 brains. TorsinA expression in En1-CKO is ablated specifically in midbrain and cerebellum. (B) Immunoblotting of torsinA expression in P5 En1-SKI CNS. ER protein calnexin is the loading control. The numbers below the torsinA immunoblot represent the relative expression of torsinA normalized to those of En1-Cre ctrl cerebral cortex. (C) Nissl and GFAP immunostaining show gliosis and cell body loss in RN and DCN. Scale bars: $500 \mu \mathrm{m}$. (D) Quantification of large neuronal perikarya in RN and DCN in the left hemispheres of P56 En1-Cre ctrl, En1-CKO, and En1-SKI mice. Med., medial DCN; Int., interposed DCN; Lat., lateral DCN. $n=3$, 2-way ANOVA. Data are shown as mean \pm SD. ${ }^{*} P<0.05 ;{ }^{* * \star} P<0.001 ;{ }^{* * * \star} P<0.0001$.

(E) Relative percentage of remaining large neuronal perikarya in RN and DCN of En1-CKO and En1-SKI. Data are converted from D.

and hind limb clasping appeared to resolve in a greater percentage of En1-SKI than En1-CKO mice (Figure 7C). In contrast to N-CKO and $\mathrm{N}$-SKI mice, En1-CKO and En1-SKI mice did not exhibit the "squinty eye" phenotype or show spontaneous hind paw twisting, so they were not easily distinguished from their littermate controls in the home cage (Figure 7C). Both mutants were impaired in the beam-walking test, exhibiting significant increases in the number of footslip/cross (Figure 7, F-I, and Supplemental Video 3). To further explore the age-related changes observed in the tail suspension test, we tested the animals on a forelimb wire-suspension/wire-hang task at multiple ages. While both mutants were significantly impaired at 2 to 3 weeks of age, En1-CKO continued to perform abnormally with maturation (Figure 7, J and L), whereas En1-SKI mice showed age-related improvement (Figure 7, K and $\mathrm{M}$ ). These data suggest that the residual function of DYT1 mutant torsinA may allow the maturing CNS to compensate to some extent. Comparison of the histopathological and behavioral phenotypes of En1-CKO and En1-SKI mice, consid- ered with biochemical studies showing loss-of-function effects of the DYT1 mutation $(9,10)$, suggests strongly that the DYT1 mutation causes neurodegeneration and dystonic-like twisting movements by impairing torsinA function during CNS maturation.

TorsinA loss of function disrupts protein quality control selectively in sensorimotor circuits. In vitro and nematode studies provide conflicting accounts of the involvement of torsinA function in protein quality control $(13,14,33-35)$. The increase in ER stress markers we observed in torsinA-sensitive sensorimotor regions (Supplemental Figure 2) suggested that loss of torsinA function may disrupt these pathways. We examined protein quality control machinery to identify potential molecular mechanisms underlying the selective insult to sensorimotor structures in torsinA mutant mice. We observed abnormal accumulation of perinuclear ubiquitin immunostaining in N-CKO mice (Figure 8, A and B, and Supplemental Figure 5A). This abnormality was largely localized to previously described torsinA-sensitive regions, though it seemed somewhat more wide- 
A

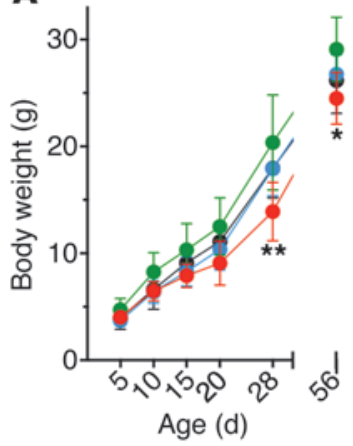

En1-CKO cohort

WT $(n=9)$

Flx-ctrl $(n=7)$

En1-Cre ctrl $(n=7)$

En1-CKO $(n=6)$

D En1-Cre crtl

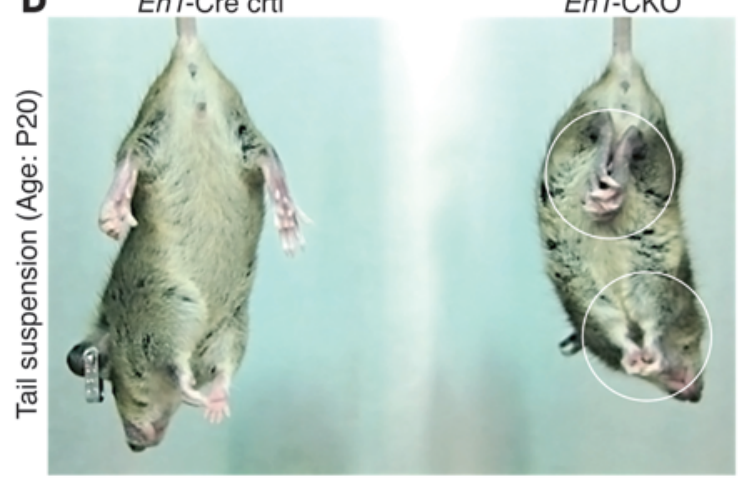

E

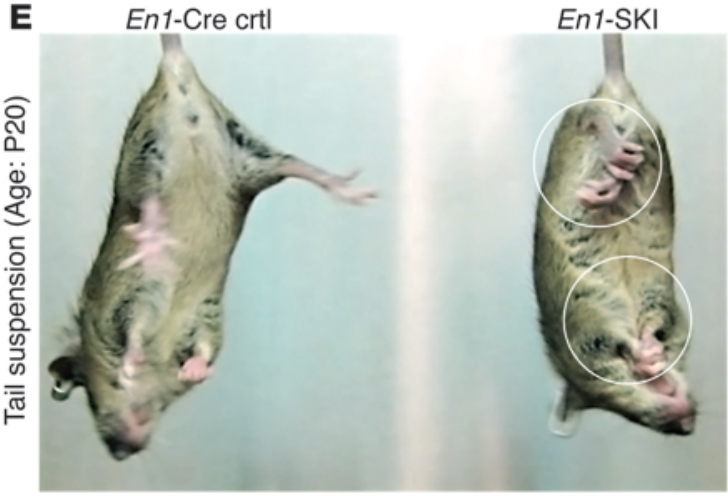

En1-SKI $(n=6)$
B

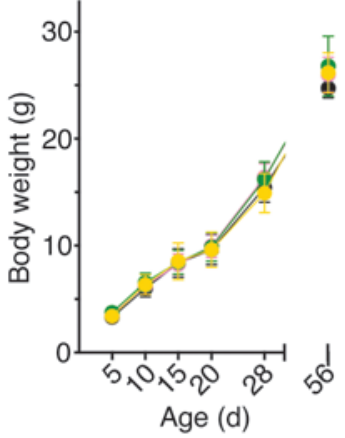

En1-SKI cohort

- WT $(n=5)$

WT $/ \Delta E(n=7)$

- En1-Cre ctrl $(n=7)$
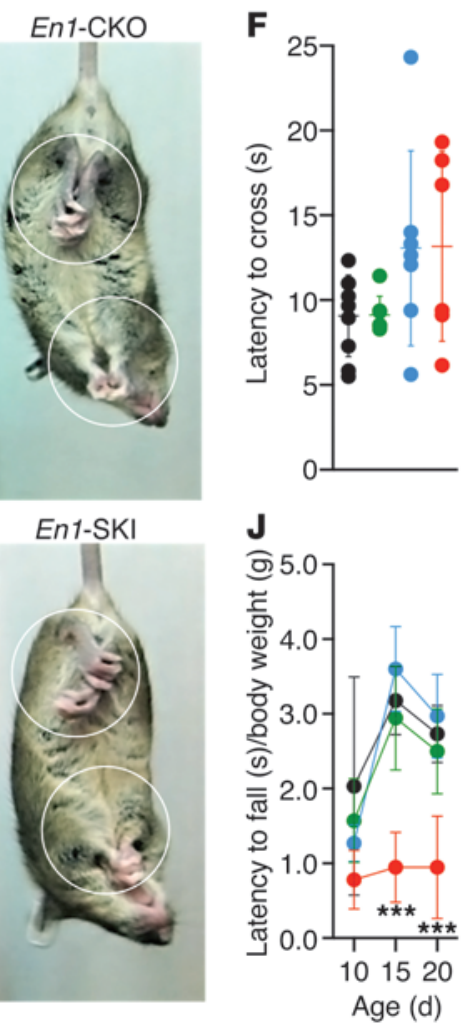

C

F

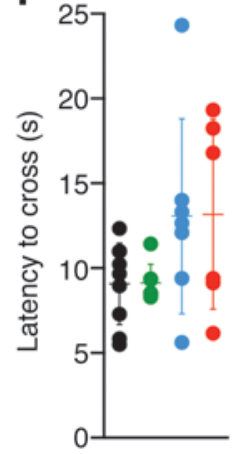

\begin{tabular}{|c|c|c|c|c|c|c} 
& Age & $\begin{array}{c}\text { Forelimb- } \\
\text { clasping }\end{array}$ & $\begin{array}{c}\text { Forepaw- } \\
\text { clenching }\end{array}$ & $\begin{array}{c}\text { Hindlimb- } \\
\text { clasping }\end{array}$ & $\begin{array}{c}\text { Twisted } \\
\text { hindpaw(s) }\end{array}$ & $\begin{array}{c}\text { Squinty } \\
\text { eye(s) }\end{array}$ \\
\hline \multirow{2}{*}{ En1-CKO } & P15 & $\begin{array}{c}100 \% \\
(9 / 9)\end{array}$ & $\begin{array}{c}100 \% \\
(9 / 9)\end{array}$ & $\begin{array}{c}100 \% \\
(9 / 9)\end{array}$ & $\begin{array}{c}0 \% \\
(0 / 9)\end{array}$ & $\begin{array}{c}0 \% \\
(0 / 9)\end{array}$ \\
\hline & P56 & $\begin{array}{c}88.89 \% \\
(8 / 9)\end{array}$ & $\begin{array}{c}88.89 \% \\
(8 / 9)\end{array}$ & $\begin{array}{c}44.44 \% \\
(4 / 9)\end{array}$ & $\begin{array}{c}0 \% \\
(0 / 9)\end{array}$ & $\begin{array}{c}0 \% \\
(0 / 9)\end{array}$ \\
\hline \multirow{2}{*}{ En1-SKI } & P15 & $\begin{array}{c}100 \% \\
(14 / 14)\end{array}$ & $\begin{array}{c}100 \% \\
(14 / 14)\end{array}$ & $\begin{array}{c}100 \% \\
(14 / 14)\end{array}$ & $\begin{array}{c}0 \% \\
(0 / 14)\end{array}$ & $\begin{array}{c}0 \% \\
(0 / 14)\end{array}$ \\
\cline { 2 - 7 } & P56 & $\begin{array}{c}71.43 \% \\
(10 / 14)\end{array}$ & $\begin{array}{c}42.86 \% \\
(6 / 14)\end{array}$ & $\begin{array}{c}14.29 \% \\
(2 / 14)\end{array}$ & $\begin{array}{c}0 \% \\
(0 / 14)\end{array}$ & $\begin{array}{c}0 \% \\
(0 / 14)\end{array}$
\end{tabular}

G

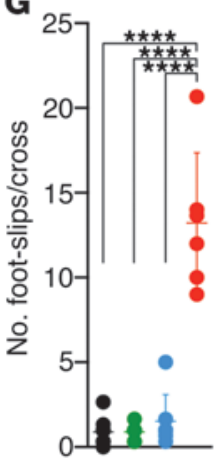

H

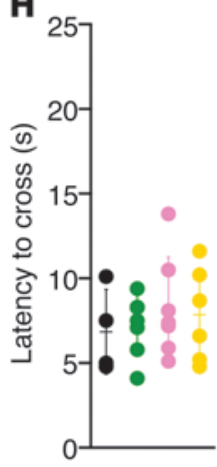

$I_{25}$

K
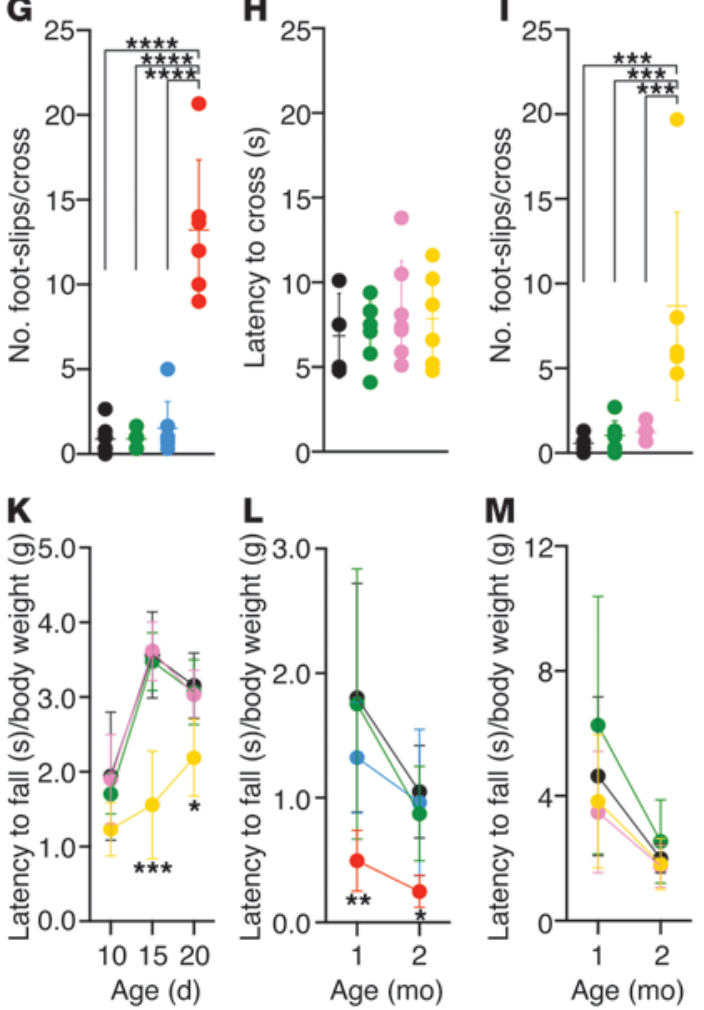

M

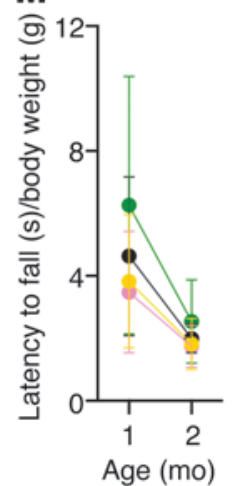

Figure 7

Characterization of behavioral abnormalities in En1-CKO and En1-SKI mice. (A) Growth curves of En1-CKO cohort. (B) Growth curves of En1SKI cohort. En1-SKI mice are significantly lighter at P28 and P56. (C) The frequency of abnormal behaviors in En1-CKO and En1-SKI mutants at P15 and P56. (D) En1-CKO and (E) En1-SKI exhibit limb clasping and forepaw clenching at P20 during the tail-hang test. (F-I) Beam walking test. Latency to cross and footslips for En1-CKO (F and $\mathbf{G})$ and En1-SKI ( $\mathbf{H}$ and $\mathbf{I})$ mice at 2 months of age. ( $\mathbf{J}$ and $\mathbf{K})$ Preweaning forelimb wiresuspension test. Latency to fall for En1-CKO (J) and En1-SKI (K) mice at different ages. (L and M) Wire-hang test. Latency to fall for En1-CKO (L) and En1-SKI (M) mice at different ages. 2-Way ANOVA. Data are shown as mean \pm SD. ${ }^{*} P<0.05 ;{ }^{* *} P<0.01 ;{ }^{* * *} P<0.001 ;{ }^{* * * *} P<0.0001$.

spread in thalamus and also showed spotty involvement of the hippocampus. Immunogold electron microscopy using an antiubiquitin antibody showed gold particles localized within the perinuclear space, in some cases within the nuclear membrane "blebs" that form in torsinA null neurons (ref. 8 and Figure 8C). Many gold particles were closely apposed to NE membranes. As torsin $\mathrm{A}$ is reported to function in the ER-associated degradation
(ERAD) pathway (13), which includes membrane-bound E3 ubiquitin ligases, we sought evidence implicating one of these molecules in the perinuclear accumulation of ubiquitin. The neuron-specific E3 ubiquitin ligase HRD1 showed abnormal perinuclear accumulation (Figure 8D and Supplemental Figure 5B) in a pattern highly similar to that seen for ubiquitin, consistent with a potential role in the ubiquitin accumulation. Perinuclear accumulation of ubiquitin 
A

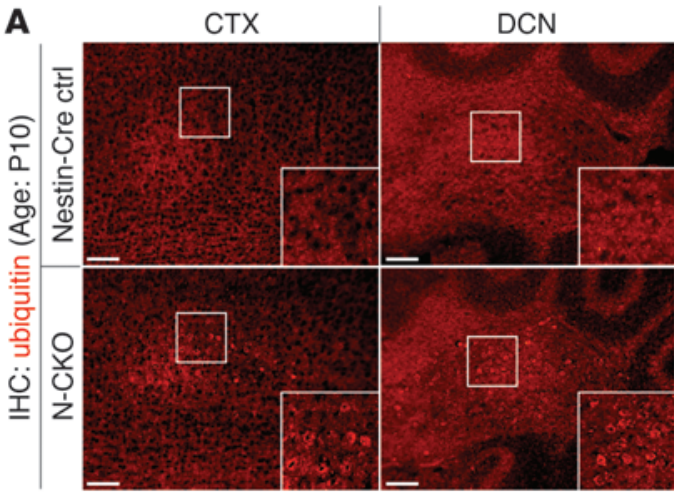

C

Immunogold: ubiquitin

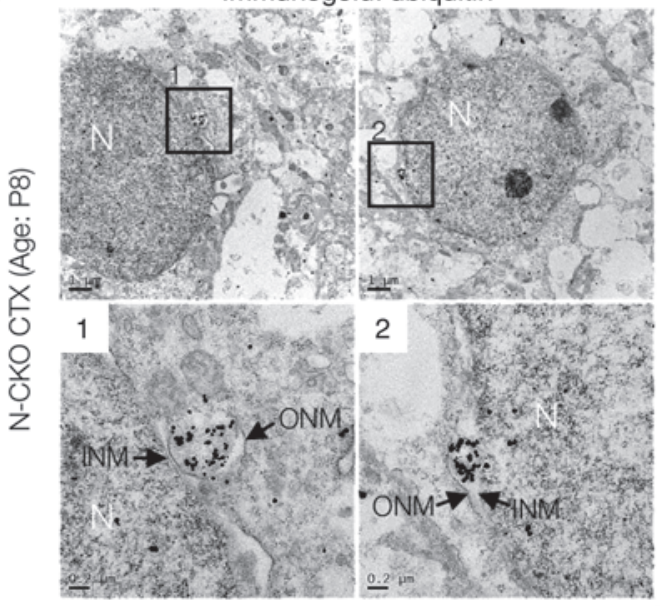

E
B

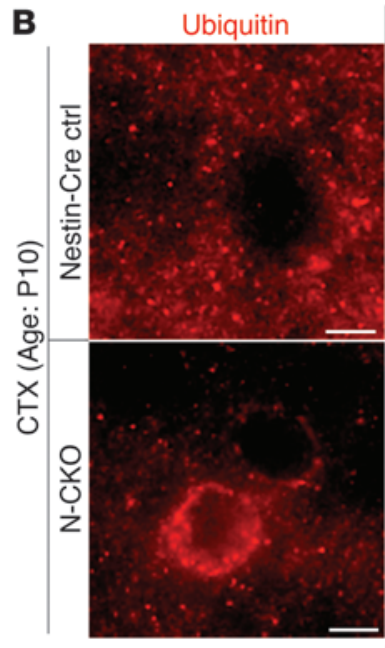

DAPI

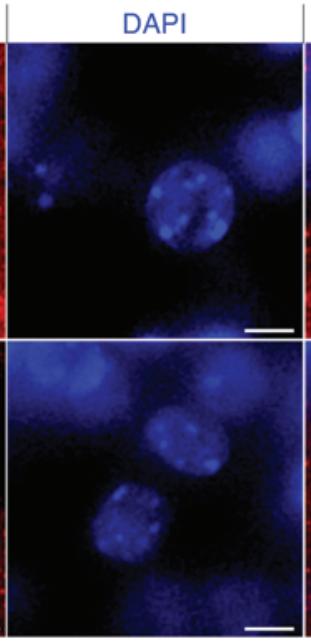

DCN
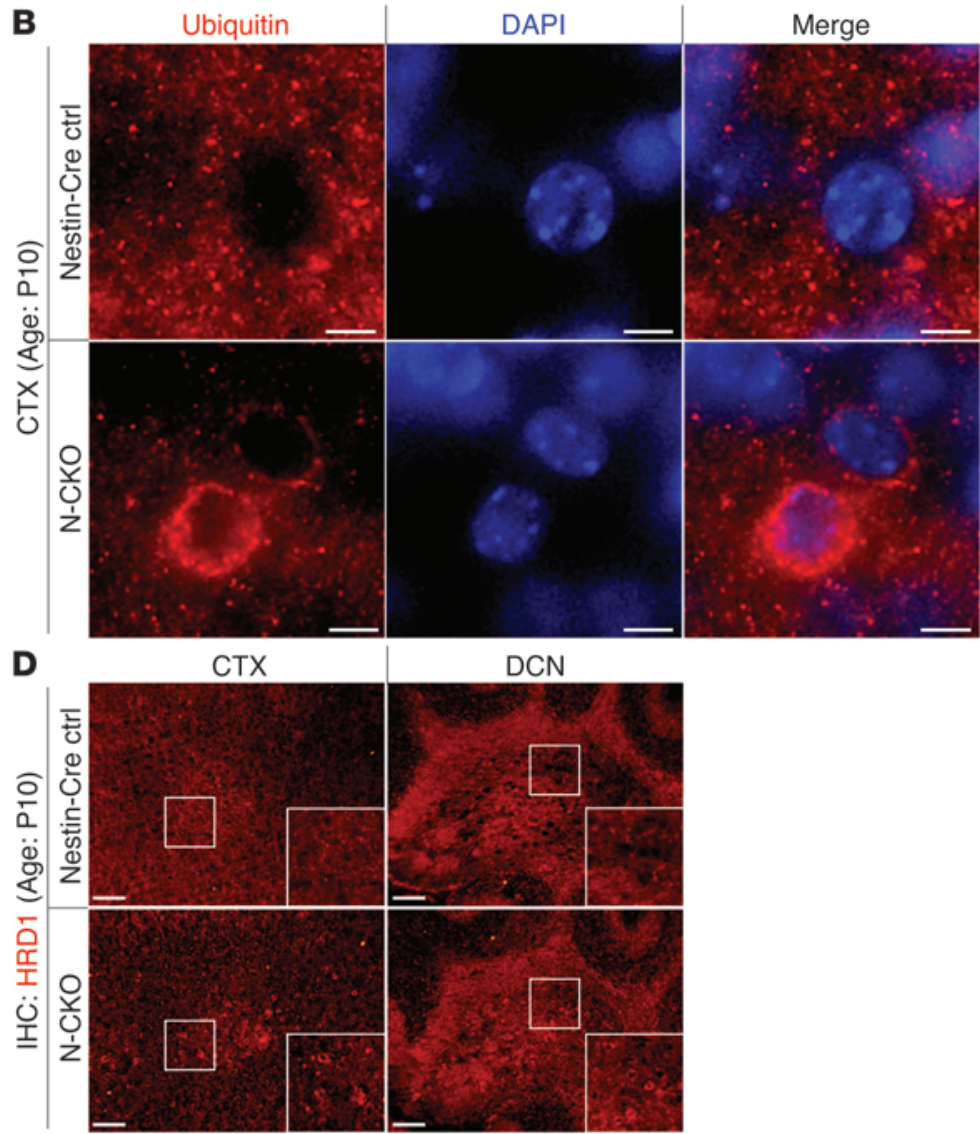

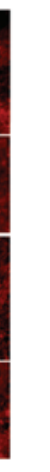

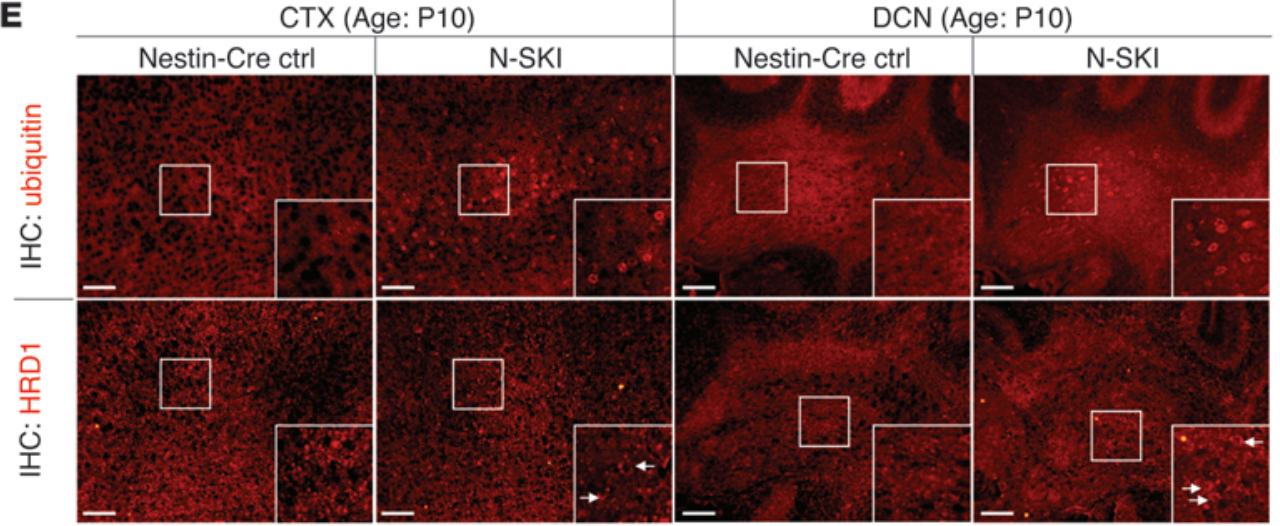

Figure 8

TorsinA loss of function causes defects in protein quality control machinery selectively in sensorimotor regions. (A) Perinuclear accumulation of ubiquitin immunoreactivity in P10 brains in cerebral cortex and in DCN. Scale bars: $100 \mu \mathrm{m}$. (B) High-magnification images show perinuclear accumulation of ubiquitin in P10 N-CKO cerebral cortex. Scale bars: $10 \mu \mathrm{m}$. (C) Transmission electron microscopy of immunogold labeling of ubiquitin in P8 cerebral cortex of N-CKO. Scale bars: $1 \mu \mathrm{m}$. High-magnification images show that ubiquitin accumulates within NE blebs in the perinuclear compartment (nos. 1 and 2). ONM, outer nuclear membrane; INM, inner nuclear membrane. Scale bars: $0.2 \mu \mathrm{m}$. (D) E3 ubiquitin ligase HRD1 immunoreactivity in P10 brains shows the abnormal perinuclear localization in the same regions exhibiting gliosis, neurodegeneration, and perinuclear ubiquitin accumulation. Scale bars: $100 \mu \mathrm{m}$. (E) Ubiquitin and HRD1 immunoreactivity in N-SKI brains (P10) shows abnormal perinuclear accumulation in a distribution similar to that seen in N-CKO mice. Scale bars: $100 \mu \mathrm{m}$.

and HRD1 was present as early as P0 (Supplemental Figure 5, C and D), including in constitutive torsinA null mice (ref. 8 and Supplemental Figure 6), indicating that these events are upstream of the previously described phenotypes (e.g., gliosis and upregulation of ER stress markers), which occur later (Supplemental Figure 2). $\mathrm{N}$-SKI mice also showed abnormalities of ubiquitin and HRD1 immunostaining (Figure 8E and Supplemental Figure 7), linking these molecular effects to the DYT1 mutation.

\section{Discussion}

Our studies establish a genetic model of primary dystonia with overtly abnormal movements, based on a cause of primary dysto- 
nia in humans. Mice modeling the dominant-negative action of the DYT1 mutation (N-SKI) feature a pattern of selective neuronal vulnerability and abnormal movements that emerge during postnatal CNS maturation, a picture broadly consistent with clinical features of the human disease. We provide evidence that by impairing torsinA function, the DYT1 mutation leads to mislocalization of the E3 ubiquitin ligase HRD1 and altered protein homeostasis, followed by subtle, age-restricted, and region-selective neurodegeneration. These events occur in a relatively discrete group of sensorimotor structures, including cortex, GP, and DCN, whose relevance to the disease is supported by DTI and functional imaging studies of human subjects with DYT1 dystonia $(32,36)$. Collectively, these findings link torsinA hypofunction to abnormal twisting movements. Our observations also raise the intriguing possibility that low levels of neurodegeneration during development, by disrupting sensorimotor circuit maturation and function, may contribute to the pathogenesis of dystonic movements.

The early age at onset and phenomenology of the abnormal movements in N-SKI mice are reminiscent of the clinical features of DYT1 dystonia. N-SKI mice appear normal initially, but begin to develop overt abnormal twisting movements during the second week of life, a time of active maturation of CNS sensorimotor circuits. As in the human disease, the severity of these abnormal movements does not continue to progress in adult animals, instead becoming relatively fixed in juvenile mice. Similar to human dystonia, these movements show action dependence, occurring or worsening with gait and during tail suspension. Not all N-SKI mice are uniformly affected, potentially modeling the variable expressivity of the DYT1 mutation (37).

Several characteristics unique to N-SKI mice may account for their novel behavioral and neuropathological phenotypes. The degree of torsinA loss of function appears to be one factor. Unlike previous conditional mutants of torsinA, the CNS-restricted Tor $1 a^{\Delta \mathrm{E} /-}$ genotype models the presumed dominant negative behavior of $\Delta \mathrm{E}$-torsin $\mathrm{A}$. This design reduces torsin $\mathrm{A}$ function to a greater extent than in heterozygous $\mathrm{KO}$ or knockin mice, which continue to express WT torsinA and do not exhibit histopathological change or overt abnormal movements $(8,27)$. The anatomic distribution and timing of torsinA loss of function also appear to be important. TorsinA hypofunction was induced prenatally and globally in the CNS of N-SKI mice, whereas in previous conditional mutants, torsinA loss of function was initiated either postnatally or in restricted brain regions or cell types (28-30). In contrast to constitutive $\mathrm{KO}$ or homozygous knockin mice that die shortly after birth, N-SKI mice survive beyond the perinatal period, enabling the events of postnatal circuit formation to proceed in the context of global and marked torsinA hypofunction. The symptoms of DYT1 dystonia typically emerge in school-aged children (ages 8-16), and mutation carriers who are unaffected by their early 20 s typically remain dystonia free for life. Considered together, these observations suggest the presence of an early critical period of sensorimotor circuit susceptibility to torsinA hypofunction that is modeled in N-SKI mice, which may explain the development of overt twisting movements.

The anatomic and temporal pattern of abnormalities observed in torsinA mutant mice indicate age- and region-specific functions for torsin A, despite its broad expression pattern in humans and rodents $(8,38,39)$. Several mechanisms may underlie these patterns of vulnerability. The exclusive occurrence of molecular and neurodegenerative changes during the first several postnatal weeks suggests that processes highly active during CNS maturation may render neurons particularly vulnerable to torsinA loss of function. This phenomenon is reminiscent of the age-sensitive vulnerability of the CNS to ethanol-induced excitotoxic cell death (40), which coincides with the period of synaptogenesis. Synaptogenesis and related aspects of postnatal CNS maturation impose considerable proteostatic stress on neurons, requiring the generation of tremendous numbers of proteins necessary to build and support synapses and convey signals, including receptors, transporters, ion channels, and structural elements. The generation of these proteins greatly increases demand for nucleocytosolic transport of mRNA and protein synthetic and quality control mechanisms. Previous work and our current observations implicate torsinA in these processes, which may fail in the face of coexistent high-demand and torsinA hypofunction. In contrast to the developmentally restricted neurodegeneration observed here, most prevalent neurodegenerative diseases (such as Parkinson and Alzheimer diseases) begin during senescence and show a propensity to relentlessly affect a wider array of brain structures over time. These differences highlight the unique features of torsinA-related neurodegeneration and illustrate how the changing vulnerability of neurons throughout the life span can participate in determining disease phenotype.

While developmental considerations appear important in explaining the temporal susceptibility to torsinA loss of function, they cannot easily account for the differences in differential vulnerability of brain regions. We previously reported that torsin A and torsinB share redundant functions, demonstrating that differences in the expression level of torsinB is a key determinant of the differential susceptibility of cells to torsinA loss of function (41). In that report, a survey of torsinB levels in different brain regions showed that hippocampus and striatum, regions that do not exhibit gliosis in $\mathrm{N}-\mathrm{CKO}$ or $\mathrm{N}-\mathrm{SKI}$ mice, express particularly high levels of torsinB. These data raise the possibility that relative levels of expression of torsinA and torsin $B$ may determine the vulnerability of a neuron to torsin $A$ hypofunction. Interestingly, the expression levels of torsin A and torsinB change considerably and inversely during postnatal development (42), suggesting that this mechanism may also contribute to the developmental-dependent aspect of torsinA loss of function.

The observation of neurodegeneration in torsinA mutant mice demonstrates a requirement for torsinA function in maintaining neuronal viability not previously reported. The abnormal accumulation of ubiquitin in a perinuclear pattern suggests a potential role for torsinA in the poorly understood processes of nuclear protein quality control. The accumulated ubiquitin most likely arises from within the nucleus and appears localized within previously described NE blebs that arise from the inner nuclear membrane $(8,41)$. These data raise the possibility that torsinA participates in the transport of ubiquitinated proteins across the NE through a membranous compartment similar to the nuclear egress pathway utilized by herpesvirus. TorsinA has been implicated in this pathway (43) and in a similar process in drosophila by which ribonucleoprotein granules are transported across the nuclear membrane (44).

The close association of ubiquitin with membrane led us to explore the potential role of membrane-bound E3 ubiquitin ligases in this process. These studies identified the neuronal specific E3 ubiquitin ligase HRD1 as a potential mediator of the perinuclear ubiquitin accumulation (45). HRD1 is part of a multiprotein complex that includes derlin, which has been reported to interact with torsinA (13). Hrd1-null mice exhibit embryonic lethality, and cells derived from these animals are more susceptible to ER stressinduced cell death (46), establishing a link between HRD1 function 
and cell viability. Neurons in which HRD1 was mislocalized were present in an anatomic pattern highly similar to that observed for perinuclear ubiquitin accumulation, gliosis, and neurodegeneration, suggesting a potential relationship among these processes. These data raise the possibilities that torsinA is involved in regulating the spatial localization of HRD1 within the ER/NE endomembrane space and that HRD1 mislocalization contributes to torsinAmediated neurodegeneration. Spatial regulation of an E3 ubiquitin ligase within the ER/NE space has been demonstrated in yeast (47), and torsin A has binding partners in both of these locations $(18,20$, 48). Indeed, one effect of the $\Delta \mathrm{E}$ mutation is to disrupt the distribution of torsinA between these 2 compartments $(7,17,19)$. Future studies will be required to clarify the relationship among abnormalities of HRD1, ubiquitin accumulation, and neurodegeneration.

The occurrence of twisting movements in N-SKI mice provided an opportunity to begin to dissect the underlying neural substrate. Clinical and experimental studies of secondary dystonia strongly implicate forebrain dysfunction as a cause of dystonia $(49,50)$, but several hindbrain elements have also been implicated (51-54). Taking advantage of different Cre-expressing lines, we began to explore which brain regions are sufficient to mimic the behavioral abnormalities of N-SKI mice. Ablation of torsinA with Emx1-Cre, which deletes in cortical glutamatergic neurons (55), recapitulated the histopathological changes in layer $\mathrm{V}$ cortex observed in $\mathrm{N}-\mathrm{CKO}$ and $\mathrm{N}$-SKI mice, but did not cause overt twisting movements either spontaneously or during tail suspension. Ablating torsinA with En1-Cre mice, which deletes broadly in midbrain and hindbrain structures, and some spinal interneurons (56), also recapitulated the histopathological changes in torsinA-sensitive structures encompassed within the Cre-expressing region. En1-SKI mice exhibited abnormal twisting movements selectively during tail suspension, indicating that loss of torsinA function in hindbrain is sufficient to cause some - but not all - of the N-SKI phenotypes. Two observations indicate that brain regions outside of the En1-SKI affected areas participate in the abnormal twisting movements in N-SKI mice. First, En1-SKI mice do not exhibit abnormal hind limb postures or twisting movements at rest or spontaneously during gait, as seen in N-SKI mice. Second, whereas the motor phenotype of N-SKI mice remains relatively fixed from 2 weeks to 2 months of age, aspects of the En1-SKI phenotype improve during this time. The abnormalities observed in the spinal cords of N-SKI mice indicate a potential role for spinal circuits in torsinA-related abnormal movements. The findings in Emx1- and En1-CKO mice do not exclude a role for the striatum or other forebrain structures in DYT1 dystonia, as the Emx1-Cre transgene expresses in only a subset of cortical neurons. Even in N-SKI mice, structures that appear normal histopathologically may lose discrete populations of cells not sufficient to elicit gliosis, and our studies are not designed to detect cellular dysfunction not manifesting as histopathological change. Indeed, the marked susceptibility of several sensorimotor structures to torsinA loss of function, together with clinical and human electrophysiological studies of dystonia (1), indicates that primary dystonia likely results from the coincident defects of or abnormal communication between multiple regions, rather than arising from a single structure.

Our findings raise new questions concerning the cellular substrate of DYT1 dystonia. Neurodegeneration is traditionally thought not to be a pathogenic process in primary dystonia, but this view is based on the postmortem analysis of very few brains from subjects with primary dystonia (4), and accumulating neuroimaging evidence suggests the presence of structural brain abnor- malities in dystonia (57), including in DYT1 dystonia (31). We sought but were unable to locate postmortem tissue of sufficient quantity or quality to carefully examine for the presence of neurodegeneration in DYT1 dystonia. The loss of modest numbers of neurons from several key sensorimotor structures could disrupt the developmental trajectory of motor circuits, leading to dystonia. Another critical element may be abnormalities of the surviving neurons, made dysfunctional by torsinA-related abnormalities of protein homeostasis. Clarification of these and related issues will require "rescue" experiments that test the necessity of specific brain regions or cell types in mediating the N-SKI phenotype.

\section{Methods}

\section{Animal busbandry and genotyping}

The Tor1a construct was built using recombineering (58). First Bac clones containing the gene of interest were ordered from CHORI. The BAC clones for Tor1a were RP23-337K9 and RP24231D2. Chimeric mice were generated using standard procedures. Following generation of chimeric mice, they were intercrossed to ROSA26-FLPe mice to remove the frt-flanked neomycin resistance gene, generating the "clean" floxed allele used for all studies. To genotype these torsinA mutants, we used the following primers: lox-gtF: CCTGCCTCAGCCTAACTACG; frt-gtF: CAACAGAAAGCCCATTTGTCT; frt-gtR: AGGCCTTGAATGACAACCAC. These primers produce the following band sizes on PCR: WT, 154 bp; KO, 343 bp; floxed allele, $250 \mathrm{bp}$. We utilized the following PCR program: $94^{\circ} \mathrm{C}, 3$ minutes; $94^{\circ} \mathrm{C}, 30$ seconds; $55^{\circ} \mathrm{C}, 30$ seconds; $72^{\circ} \mathrm{C}, 30$ seconds, 33 cycles; $72^{\circ} \mathrm{C}, 5$ minutes. The breeding strategy used to generate conditional null animals was as follows: Tor $1 \mathrm{a}^{+/-}$ $\mathrm{Cre}^{+} \times$Torlaflox/flox. This breeding strategy produced the following offspring: Tor 1 $a^{f l o x /+}$ (WT); Tor1 $a^{\text {flox/ } /-}$ (Flx ctrl); Tor1 $a^{f l o x /+} \mathrm{Cre}^{+}$(Cre ctrl); and Tor1 $a^{f l x /-}$ $\mathrm{Cre}^{+}(\mathrm{CKO})$. The breeding strategy to produce SKI animals was as follows: $\operatorname{Tor} 1 a^{+/ \Delta E} \mathrm{Cre}^{+} \times \operatorname{Tor} 1 a^{f l o x /+}$. This breeding strategy produced the following off-

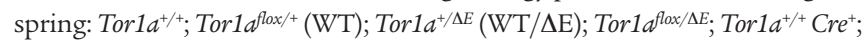

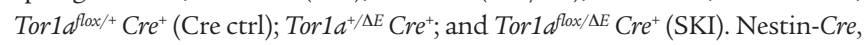
En1-Cre, and Emx1-Cre mice were obtained from The Jackson Laboratory.

\section{Histology and immunohistochemistry}

All mice were perfused and brains were fixed in 4\% PFA for 24 hours followed by dehydration in $30 \%$ PBS-buffered sucrose solution for 48 hours at $4{ }^{\circ} \mathrm{C}$. For all analyses, $30-\mu \mathrm{m}$ tissue sections from control and mutant mice were collected and processed in parallel on the same slides. Unstained tissue sections were permeabilized using PBS containing 0.3\% Triton X-100 followed by blocking in $10 \%$ normal donkey serum for 1 hour at room temperature (RT). The sections were incubated with primary antibodies at $4{ }^{\circ} \mathrm{C}$ overnight. To visualize the proteins of interest, the tissue sections were incubated with either fluorophore-conjugated (Dylight-488 and Dylight-550) or biotinylated-conjugated secondary antibodies (1:1000 and 1:200 dilution, respectively; Jackson ImmunoResearch Laboratories Inc.) for 2 hours at RT. After washing with $0.25 \%$ TBST, VECTASTAIN Elite ABC system (VECTOR Laboratories Inc.) and chromogen DAB (Sigma-Aldrich) were applied if necessary. At the last step, the sections were counterstained using either DAPI $(0.1 \mu \mathrm{g} / \mathrm{ml})$, or Methyl green (Sigma-Aldrich). To determine the number of AC3-positive cells in brain tissues, 4 right hemispheres from each genotype were serially cryosectioned from medial to lateral and stained with cleaved caspase-3 (1:100; Cell Signaling). One-third of the total sections were sampled for counting the AC3-positive nuclei. The primary antibodies used in this report are as follows: torsinA (1:100, Abcam), NeuN (1:1000, Millipore), GFAP (1:1000, Dako), ubiquitin (1:200, Dako), cleaved caspase-3 (1:100, Cell Signaling), CHOP (1:100, Cell Signaling), phospho-eIF2 $\alpha$ (Ser-51) (1:100, Cell Signaling), phospho-JNK (T183/Y185) (1:100, Cell Signaling), 
and HRD1 (1:100, Sigma-Aldrich). Histological examination and digital photography were carried out under a Axioskop2 microscope (Carl Zeiss Microscopy). The images were captured with an Olympus digital camera (model DP70) using DP Controller software (Version 1.2.1.10 8).

\section{Protein extraction, SDS-PAGE, and Western blotting}

The brain homogenates were prepared from $\mathrm{P0}$ (N-CKO and N-SKI) or P5 (En1-CKO and En1-SKI) mouse brain tissues using 1\% NP-40 lysis buffer supplemented with protease inhibitors (Complete Mini Protease inhibitors, Roche), and each sample aliquot was denatured at $95^{\circ} \mathrm{C}$ for 5 minutes in $1 \times$ SDS sample buffer (1 mM Tris-HCl, pH 6.8; $25 \%$ glycerol; $2 \%$ SDS; $0.1 \%$ bromphenol blue; $5 \% \beta$-mercaptoethanol). The homogenate samples were subjected to $10 \%$ SDS-PAGE followed by the protein transfer onto nitrocellulose membranes. The membranes were incubated with antibodies against either torsinA (1:5000) or calnexin (1:10000) for 1 hour at RT. After washing several times with $0.25 \%$ TBST, the membranes were incubated with horseradish peroxidase-conjugated secondary antibodies (1:5000, Cell Signaling) to detect torsinA or calnexin immunoreactivity.

\section{Cell counting of RN and DCN}

To determine the number of $\mathrm{RN}$ and $\mathrm{DCN}$ neurons, 3 left hemispheres from each genotype were serially cryosectioned to include the entire rostrocaudal extent of both nuclei. All tissue sections were processed with Nissl counterstain, and one-third of the sections covering each subgroup were sampled for the cell counts with an automatic stereology system (newCAST, Visiopharm) on an Olympus BX51 microscope (Leica Microsystems, Germany) using the Optical Fractionator. All cell counts were performed with a $\times 60$ objective by an investigator blind to genotype. The counting fraction covered $50 \%$ of each subgroup. The RN and DCN were identified using established anatomical landmarks (corresponding to the atlas of Franklin and Paxinos, ref. 59). Criteria for the identification of neurons were as follows: soma size, $25 \mu \mathrm{m}$ or greater, cytoplasm with prominent Nissl substance and clearly visible nucleolus.

\section{Behavioral testing}

Mice of different genotypes were housed together to avoid biasing based on environment. Only male mice were used for behavioral testing. The twisted paw and eye-opening phenotype were assessed by monitoring animals in their home cages for 5 minutes at P21 and P56 (N-SKI, Supplemental Figure 4B) or at P15 and P56 (En1-CKO and En1-SKI, Figure 7C). Other behavioral tests were performed as below.

Tail hang. Each mouse was suspended by its tail and videotaped for 30 seconds. The presence or absence of limb clasping or paw clenching was assessed during this 30 seconds. Limb clasping was defined as sustained abnormal posture for 2 seconds or more, with the paw either forcefully extended in the air or around the tail or held tightly against the body. Paw clenching was defined as the clenching of all toes of the front paw or paws. TorsinA mutant animals were also observed as exhibiting abnormalappearing truncal movements, but these movements were not quantified.

Preweaning forelimb wire suspension. As previously described, with subtle modification (60), each mouse was made to grasp with their forepaws a $3 \mathrm{~mm}$ wire elevated $20 \mathrm{~cm}$ above the table top. The latency to fall of each animal was recorded in every trial. The cut-off time for each trial was 30 seconds. The performance for each mouse was calculated by taking the average of 5 trials.

Wire hang. As previously described, with subtle modification (61), each mouse was placed on an elevated wire cage top, which was then inverted and suspended at $20 \mathrm{~cm}$ above the table top. The latency to fall of each mouse was recorded for every trial. The cut-off time for each trial was 5 minutes. The performance for each animal was calculated by taking the average of 5 trials.

Beam walking. As previously described, with subtle modification (62), each mouse was trained to cross a $50-\mathrm{cm}$ long $5-\mathrm{mm}$ square plastic bar.
Training consisted of 4 trials/day for 3 consecutive days. At the start of each trial, the mouse was placed on a clear open platform; a dark box at the opposite end of the bar provided the motivation for the mouse to cross the beam. These trials were videotaped, and the time to cross and number of foot slips were subsequently determined by an investigator blinded to genotype. An animal's performance was calculated by taking the average of the values obtained over 3 trials on the fourth day.

\section{Immunogold electron microscopy}

Immunoelectron microscopy experiments were performed on P8 cerebral cortices of Tor $1 a^{f l o x /+}$; nestin-Cre (nestin-Cre ctrl) and N-CKO mice. The mice were perfused with $4 \% \mathrm{PFA}$ and $0.2 \%$ glutaraldehyde in $0.1 \mathrm{M}$ phosphate buffer ( $\mathrm{pH} 7.4$ ). The tissue sections were collected at $50 \mu \mathrm{m}$ using a Vibratome (Technical Products International) and incubated with $0.1 \%$ $\mathrm{NaBH}_{4}$ in $0.1 \mathrm{M} \mathrm{PB}$ for aldehyde inactivation. After permeabilizing with $0.05 \%$ Triton $\mathrm{X}-100$ in $0.1 \mathrm{M}$ PB for 30 minutes, the sections were incubated with $5 \%$ BSA, $0.1 \%$ CWFS gelatin, and $5 \%$ normal goat serum in PBS for 1 hour at $4{ }^{\circ} \mathrm{C}$. The ubiquitin antibody (1:200) was diluted in PBS/ BSA-c (Electron Microscopy Sciences) and incubated with the sections at $4{ }^{\circ} \mathrm{C}$ for 24 hours. After washing with PBS/BSA-c, the tissue sections were incubated with Ultrasmall Gold Conjugates (Electron Microscopy Sciences) diluted 1:100 in PBS/BSA-c for 16 hours at $4{ }^{\circ} \mathrm{C}$. Following incubation, the sections were washed with ECS (Electron Microscopy Sciences) for preenhancement. R-gent SE-EM (Electron Microscopy Sciences) was applied to the sections for silver enhancement, and $0.03 \mathrm{M} \mathrm{Na}_{2} \mathrm{~S}_{2} \mathrm{O}_{3}$ in ECS was used for enhancement termination. At the final step, the sections were post-fixed with $2.5 \%$ glutaraldehyde in $0.1 \mathrm{M} \mathrm{PB}$ for 2 hours followed by Osmium fixation using $0.5 \% \mathrm{OsO}_{4}$ in $0.1 \mathrm{M} \mathrm{PB}$ for $30 \mathrm{~min}$ utes prior to dehydration and embedding. Histological examination and digital photography were performed on a Philips CM-100 TEM (Philips/ FEI Corporation). The images were captured using AMTV540 (Advanced Microscopy Techniques).

\section{Statistics}

Statistical analysis was performed using GraphPad Prism 5 (GraphPad Software Inc.). Data are presented as mean SD. Statistical significance of differences between groups was evaluated using 2-way ANOVA with posthoc Bonferroni's test.

\section{Study approval}

All animal protocols were approved by the University of Michigan University Committee on the Use and Care of Animals (UCUCa).

\section{Acknowledgments}

We thank Roger L. Albin, Andrew P. Lieberman, Miriam H. Meisler, Roman J. Giger, and members of the Dauer lab for their careful reading of this manuscript and helpful comments. This research project was supported in part by Bachmann-Strauss Dystonia and Parkinson Disease Foundation, a Fellowship from the Dystonia Medical Research Foundation (to L.M. Tanabe), the Robert P. Apkarian Integrated Electron Microscopy Core of Emory University, and a grant from the National Institute of Neurological Disorders and Stroke (1R01NS077730 to W.T. Dauer).

Received for publication August 21, 2013, and accepted in revised form April 10, 2014.

Address correspondence to: William T. Dauer, 109 Zina Pitcher Place, BSRB Room 4003, Ann Arbor, Michigan 48109-2200, USA. Phone: 734.615.3874; Fax: 734.647.9777; E-mail: dauer@med.umich.edu. 
1. Tanabe LM, Kim CE, Alagem N, Dauer WT. Primary dystonia: molecules and mechanisms. Nat Rev Neurol. 2009;5(11):598-609.

2. Ozelius LJ, Bressman SB. Genetic and clinical features of primary torsion dystonia. Neurobiol Dis. 2011;42(2):127-135.

3. Albanese A, et al. Phenomenology and classification of dystonia: a consensus update. Mov Disord. 2013; 28(7):863-873.

4. Standaert DG. Update on the pathology of dystonia. Neurobiol Dis. 2011;42(2):148-151.

5 . Hewett J, et al. Mutant torsinA, responsible for early-onset torsion dystonia, forms membrane inclusions in cultured neural cells. Hum Mol Genet. 2000; 9(9):1403-1413.

6. Kustedjo K, Bracey MH, Cravatt BF. Torsin A and its torsion dystonia-associated mutant forms are lumenal glycoproteins that exhibit distinct subcellular localizations. J Biol Chem. 2000; 275(36):27933-27939.

7. Goodchild RE, Dauer WT. Mislocalization to the nuclear envelope: an effect of the dystonia-causing torsinA mutation. Proc Natl Acad Sci U S A. 2004; 101(3):847-852.

8. Goodchild RE, Kim CE, Dauer WT. Loss of the dystonia-associated protein torsinA selectively disrupts the neuronal nuclear envelope. Neuron. 2005; 48(6):923-932.

9. Naismith TV, Dalal S, Hanson PI. Interaction of torsinA with its major binding partners is impaired by the dystonia-associated DeltaGAG deletion. J Biol Chem. 2009;284(41):27866-27874.

10. Zhao C, Brown RSH, Chase AR, Eisele MR, Schlieker C. Regulation of Torsin ATPases by LAP1 and LULL1. Proc Natl Acad Sci U S A. 2013; 110(17):E1545-E1554.

11. Gonzalez-Alegre P, Bode N, Davidson BL, Paulson HL. Silencing primary dystonia: lentiviral-mediated RNA interference therapy for DYT1 dystonia. J Neurosci. 2005;25(45):10502-10509.

12. Torres GE, Sweeney AL, Beaulieu J-M, Shashidharan P, Caron MG. Effect of torsinA on membrane proteins reveals a loss of function and a dominant-negative phenotype of the dystoniaassociated DeltaE-torsinA mutant. Proc Natl Acad Sci U S A. 2004;101(44):15650-15655.

13. Nery FC, et al. TorsinA participates in endoplasmic reticulum-associated degradation. Nat Commun. 2011;2:393.

14. Chen $\mathrm{P}$, et al. The early-onset torsion dystonia-associated protein, torsin A, is a homeostatic regulator of endoplasmic reticulum stress response. Hum Mol Genet. 2010;19(18):3502-3515.

15. Hewett JW, et al. siRNA knock-down of mutant tor$\sin$ A restores processing through secretory pathway in DYT1 dystonia cells. Hum Mol Genet. 2008; 17(10):1436-1445

16. Hewett JW, et al. Mutant torsinA interferes with protein processing through the secretory pathway in DYT1 dystonia cells. Proc Natl Acad Sci US A. 2007; 104(17):7271-7276.

17. Gonzalez-Alegre P, Paulson HL. Aberrant cellular behavior of mutant torsinA implicates nuclear envelope dysfunction in DYT1 dystonia. J Neurosci. 2004 24(11):2593-2601

18. Goodchild RE, Dauer WT. The AAA+ protein tor$\sin \mathrm{A}$ interacts with a conserved domain present in LAP1 and a novel ER protein. J Cell Biol. 2005; 168(6):855-862.

19. Naismith TV, Heuser JE, Breakefield XO, Hanson PI. TorsinA in the nuclear envelope. Proc Natl Acad Sci US A. 2004;101(20):7612-7617.

20. Jungwirth MT, Kumar D, Jeong DY, Goodchild RE The nuclear envelope localization of DYT1 dysto- nia torsin $\mathrm{A}-\Delta \mathrm{E}$ requires the SUN1 LINC complex component. BMC Cell Biol. 2011;12:24.

21. Nery FC, et al. TorsinA binds the KASH domain of nesprins and participates in linkage between nuclear envelope and cytoskeleton. J Cell Sci. 2008; 121 (pt 20):3476-3486.

22. Grundmann $\mathrm{K}$, et al. Overexpression of human wildtype torsinA and human DeltaGAG torsinA in a transgenic mouse model causes phenotypic abnormalities. Neurobiol Dis. 2007;27(2):190-206.

23. Sharma $\mathrm{N}$, et al. Impaired motor learning in mice expressing torsinA with the DYT1 dystonia mutation. J Neurosci. 2005;25(22):5351-5355.

24. Page ME, et al. Cell-autonomous alteration of dopaminergic transmission by wild type and mutant (DeltaE) TorsinA in transgenic mice. Neurobiol Dis. 2010;39(3):318-326.

25 . Grundmann K, et al. Generation of a novel rodent model for DYT1 dystonia. Neurobiol Dis. 2012; 47(1):61-74.

26. Dang MT, et al. Generation and characterization of Dyt1 DeltaGAG knock-in mouse as a model for earlyonset dystonia. Exp Neurol. 2005;196(2):452-463.

27. Tanabe LM, Martin C, Dauer WT. Genetic background modulates the phenotype of a mouse model of DYT1 dystonia. PLoS One. 2012; 7(2):e32245.

28. Sciamanna $G$, et al. Cholinergic dysregulation produced by selective inactivation of the dystoniaassociated protein torsinA. Neurobiol Dis. 2012 47(3):416-427.

29. Yokoi F, Dang MT, Li J, Standaert DG, Li Y. Motor deficits and decreased striatal dopamine receptor 2 binding activity in the striatum-specific Dyt 1 conditional knockout mice. PLoS One. 2011; 6(9):e24539.

30. Zhang L, et al. Altered dendritic morphology of Purkinje cells in Dyt $1 \triangle$ GAG knock-in and purkinje cell-specific Dyt 1 conditional knockout mice. PLoS One. 2011;6(3):e18357.

31. Ulug AM, et al. Cerebellothalamocortical pathway abnormalities in torsinA DYT1 knock-in mice. Proc Natl Acad Sci U S A. 2011;108(16):6638-6643.

32. Argyelan M, et al. Cerebellothalamocortical connectivity regulates penetrance in dystonia. J Neurosci. 2009;29(31):9740-9747.

33. Gordon KL, Glenn KA, Gonzalez-Alegre P. Exploring the influence of torsinA expression on protein quality control. Neurochem Res. 2011;36(3):452-459.

34. Valastyan JS, Lindquist S. TorsinA and the torsin Ainteracting protein printor have no impact on endoplasmic reticulum stress or protein trafficking in yeast. PLoS One. 2011;6(7):e22744.

35. Burdette AJ, Churchill PF, Caldwell GA, Caldwell KA. The early-onset torsion dystonia-associated protein, torsinA, displays molecular chaperone activity in vitro. Cell Stress Chaperones. 2010; 15(5):605-617.

36. Eidelberg D, et al. The metabolic topography of idiopathic torsion dystonia. Brain. 1995; 118(pt 6):1473-1484.

37. Bressman SB, et al. Dystonia in Ashkenazi Jews: clinical characterization of a founder mutation. Ann Neurol. 1994;36(5):771-777.

38. Augood SJ, et al. Distribution of the mRNAs encoding torsin A and torsinB in the normal adult human brain. Ann Neurol. 1999;46(5):761-769.

39. Ziefer $\mathrm{P}$, et al. Molecular cloning and expression of rat torsin A in the normal and genetically dystonic $(\mathrm{dt})$ rat. Brain Res Mol Brain Res. 2002;101(1-2):132-135.

40. Ikonomidou C, et al. Ethanol-induced apoptotic neurodegeneration and fetal alcohol syndrome. Science. 2000;287(5455):1056-1060.

41. Kim CE, Perez A, Perkins G, Ellisman MH, Dauer
WT. A molecular mechanism underlying the neural-specific defect in torsinA mutant mice. Proc Natl Acad Sci U S A. 2010;107(21):9861-9866.

42. Vasudevan A, Breakefield XO, Bhide PG. Developmental patterns of torsinA and torsinB expression. Brain Res. 2006;1073-1074:139-145.

43. Maric M, et al. A functional role for TorsinA in herpes simplex virus 1 nuclear egress. J Virol. 2011; 85(19):9667-9679.

44. Jokhi $\mathrm{V}$, et al. Torsin mediates primary envelopment of large ribonucleoprotein granules at the nuclear envelope. Cell Rep. 2013;3(4):988-995.

45. Omura T, Kaneko M, Tabei N, Okuma Y, Nomura Y. Immunohistochemical localization of a ubiquitin ligase HRD1 in murine brain. J Neurosci Res. 2008; 86(7):1577-1587.

46. Yagishita N, et al. Essential role of synoviolin in embryogenesis. J Biol Chem. 2005;280(9):7909-7916.

47. Deng M, Hochstrasser M. Spatially regulated ubiquitin ligation by an ER/nuclear membrane ligase. Nature. 2006;443(7113):827-831.

48. Giles LM, Li L, Chin LS. Printor, a novel torsinAinteracting protein implicated in dystonia pathogenesis. J Biol Chem. 2009;284(32):21765-21775.

49. Bhatia KP, Marsden CD. The behavioural and motor consequences of focal lesions of the basal ganglia in man. Brain. 1994;117(pt 4):859-876.

50. Lee MS, Marsden CD. Movement disorders following lesions of the thalamus or subthalamic region. Mov Disord. 1994;9(5):493-507.

51. Raike RS, et al. Limited regional cerebellar dysfunction induces focal dystonia in mice. Neurobiol Dis. 2012;49C:200-210.

52. Calderon DP, Fremont R, Kraenzlin F, Khodakhah $\mathrm{K}$. The neural substrates of rapid-onset DystoniaParkinsonism. Nat Neurosci. 2011;14(3):357-365.

53. Stratton SE, Lorden JF. Effect of harmaline on cells of the inferior olive in the absence of tremor: differential response of genetically dystonic and harmaline-tolerant rats. Neuroscience. 1991; 41(2-3):543-549.

54. LeDoux MS, Lorden JF, Ervin JM. Cerebellectomy eliminates the motor syndrome of the genetically dystonic rat. Exp Neurol. 1993;120(2):302-310.

55. Gorski JA, et al. Cortical excitatory neurons and glia, but not GABAergic neurons, are produced in the Emx1-expressing lineage. J Neurosci. 2002; 22(15):6309-6314.

56. Wenner P, O'Donovan MJ, Matise MP. Topographi$\mathrm{cal}$ and physiological characterization of interneurons that express engrailed- 1 in the embryonic chick spinal cord. J Neurophysiol. 2000;84(5):2651-2657.

57. Ramdhani RA, Simonyan K. Primary dystonia: conceptualizing the disorder through a structural brain imaging lens. Tremor Other Hyperkinet Mov (NY). 2013;3.pii:tre-03-152-3638-4.

58. Liu P, Jenkins NA, Copeland NG. A highly efficient recombineering-based method for generating conditional knockout mutations. Genome Res. 2003; 13(3):476-484.

59. Paxinos G, Franklin KBJ. The Mouse Brain in Stereotaxic Coordinates. 3rd ed. San Diego, California, USA: Academic Press; 2008:

60. Santos M, Silva-Fernandes A, Oliveira P, Sousa N, Maciel P. Evidence for abnormal early development in a mouse model of Rett syndrome. Genes Brain Behav. 2007;6(3):277-286.

61. Sango K, et al. Mice lacking both subunits of lysosomal $\beta$-hexosaminidase display gangliosidosis and mucopolysaccharidosis. Nat Genet. 1996; 14(3):348-352.

62. Carter RJ, Morton J, Dunnett SB. Motor coordination and balance in rodents. Curr Protoc Neurosci. 2001; Chapter 8:Unit 8.12. 